\title{
Analytic Potential Energy Functions for Aluminum Clusters
}

\author{
Ahren W. Jasper, ${ }^{\dagger}$ Przemysław Staszewski, ${ }^{\ddagger}, \S$ Grażyna Staszewska, ${ }^{\ddagger, l}$ Nathan E. Schultz, ${ }^{\dagger}$ and \\ Donald G. Truhlar*, $\dagger$ \\ Department of Chemistry and Supercomputing Institute, University of Minnesota, \\ Minneapolis, Minnesota 55455-0431, Department of Theoretical Foundations of Biomedical Sciences and \\ Medical Informatics, Ludwik Rydygier Medical University, ul. Jagiellońska 13, 85-067 Bydgoszcz, Poland, and \\ Institute of Physics, Nicholas Copernicus University, ul. Grudziagdzka 5, 87-100 Torun, Poland
}

Received: February 24, 2004

\begin{abstract}
Nineteen analytic potential energy functions (PEFs) for aluminum (three pairwise additive ones, six nonpairwise additive ones with three-body terms, and ten embedded atom-type PEFs) were obtained from the literature. The PEFs were tested and reparametrized using a diverse training set that includes 20 potential energy curves and a total of 224 geometries for five aluminum clusters $\mathrm{Al}_{N}(N=2,3,4,7$, and 13) computed using hybrid density functional theory, as well as the experimental face-centered cubic cohesive energy and lattice constant. The best PEFs from the literature have mean unsigned errors (MUEs) over the clusters in the data set of $\sim 0.12 \mathrm{eV} /$ atom. The best reparametrized PEFs from the literature have MUEs of $0.06 \mathrm{eV} /$ atom. The data set is also used to develop, parametrize, and systematically study the effectiveness of several functional forms designed specifically to model many-body effects in clusters, including bond angle, screening, and coordination number effects; a total of eighteen new PEFs are proposed and tested. The best potential overall has an MUE of $0.05 \mathrm{eV} /$ atom, explicitly includes screening and coordination number effects, features linear scaling, and incorporates the accurate two-body and bulk limits.
\end{abstract}

\section{Introduction}

In a molecular dynamics simulation, a set of atomic coordinates or a wave function evolves in time according to Newton's or Schrödinger's equation of motion. Within the BornOppenheimer approximation, ${ }^{1,2}$ all of the relevant information for such a calculation is contained in the masses of the atoms and in the potential energy function (PEF), which describes the variation of the ground-state electronic energy as a function of the nuclear coordinates. The evaluation of the accuracy of a $\mathrm{PEF}$ is therefore a prerequisite for interpreting the results of any dynamical calculation in which it is used.

Numerous methods exist for computing PEFs for metallic systems and provide varying compromises of accuracy and computational affordability. For aluminum systems containing up to several hundred atoms, the PEF may be calculated using quantum mechanical methods such as hybrid density functional theory ${ }^{3,4}$ (HDFT) with an accurate basis set for small systems ( $<20$ atoms), HDFT with effective core potentials ${ }^{5-9}$ for systems containing up to $\sim 100$ atoms, and tight-binding methods ${ }^{10}$ for larger systems. For still larger systems, such as those used for studying the mechanical properties of nanostructured materials, simulations often contain at least 100 structural units of the material, i.e., millions or even billions of atoms. Simulations of this size require PEFs that may be evaluated rapidly and inexpensively, such as analytic PEFs. Inexpensive PEFs are also important for simulations of smaller systems when modeling processes with long time scales or sampling various initial conditions, temperatures, pressures, etc.

\footnotetext{
* Corresponding author. E-mail: truhlar@umn.edu.

† University of Minnesota and Supercomputing Institute.

$\doteqdot$ Visiting researcher, Department of Chemistry, University of Minnesota, 2002-2003.

$\S$ Ludwik Rydygier Medical University.

"Nicholas Copernicus University.
}

Analytic PEFs may be developed and justified in a variety of ways, but for large systems they are most often validated by comparing the results of a simulation with some set of known (e.g., experimental) results. This method is often indirect, and it may not be clear whether the success or failure of a PEF is due to the PEF itself, the dynamical method, or uncertainties in the experimental situation. More importantly, it is desirable to develop PEFs that may be used to simulate events that cannot be easily observed in the laboratory or that are not yet well understood, and it is quite possible that there are no experimental data available that are sensitive to the full domain of the PEF that is required to simulate such processes. A second, more direct method for validating PEFs involves comparing the energies and forces predicted by a PEF over a range of geometries representative of the simulation of interest with the results of electronic structure calculations. It is often difficult to determine beforehand which configurations will be important for a simulation, and large numbers of expensive calculations using high-level electronic structure calculations may be required to sample the important geometries sufficiently. Furthermore, it is often not clear if even high-level electronic structure calculations are themselves accurate, especially for systems involving metals.

The goal of this paper is to test and develop analytic PEFs for aluminum clusters using the second, more direct approach. We have previously developed ${ }^{11}$ a data set of accurate aluminum cluster energies using the PBEO hybrid density functional theory ${ }^{12-14}$ (HDFT) with the MG3 basis set, ${ }^{15,16}$ which was found to be the most accurate of several HDFT methods tested for small aluminum clusters. ${ }^{11}$ The data set has been augmented slightly in the present work, and the augmented set includes 224 geometries for $\mathrm{Al}_{N}$ clusters with $N=2,4,5,7$, and 13 .

A total of nineteen analytic PEFs that have previously been developed for aluminum have been collected from the literature. 
We have attempted to include well-tested PEFs that feature various theoretical models and parametrization schemes. First, the collected PEFs are evaluated using the aluminum cluster data set along with the experimental face centered cubic (FCC) cohesive energy and lattice constant. Next, we evaluate the relative applicability and flexibility of the various functional forms themselves by reparametrizing the PEFs against the aluminum cluster data set and the experimental cohesive energy and lattice constant using a microgenetic ${ }^{17,18}$ algorithm.

Another goal of the present paper is to systematically study many-body effects in aluminum clusters and test several functional forms, not previously applied to aluminum, that were designed specifically to model these effects.

The paper is organized as follows. Section II summarizes the PBE0/MG3 aluminum cluster data set as well as the methods used to evaluate the PEFs and optimize their parameters. Section III summarizes the analytic PEFs obtained from the literature and several additional functional forms that we designed to model many-body effects in metal clusters. Section IV contains results and discussion, and section $\mathrm{V}$ is a summary.

\section{PEF Evaluation and Optimization}

The test set is composed of several computed aluminum cluster energies as well as the experimental FCC cohesive energy and lattice constant. In a previous paper, ${ }^{11}$ the PBE0 hybrid density functional theory ${ }^{12-14}$ (HDFT) method with the MG3 basis $\operatorname{set}^{15,16}$ was validated for aluminum clusters by comparing the results of several HDFT methods with more accurate calculations for a test set of small cluster energies. The PBE0/MG3 method was found to be the most accurate of several HDFT methods tested with an MUE of only $0.01 \mathrm{eV} /$ atom averaged over a data set of small clusters. ${ }^{11}$ The PBE0/MG3 method was then used ${ }^{11}$ to develop a data set of energies for 190 geometries, including five cluster sizes $\mathrm{Al}_{N_{k}}$, where $N_{k}=$ $2,3,4,7$, and 13 for $k=1, \ldots, 5$, respectively. The data set has been augmented slightly in the present work with 34 additional $\mathrm{Al}_{2}$ calculations for a total of 224 energies.

Also considered in the evaluation of the PEFs are the bulk cohesive energies $E_{\mathrm{b}}$ for an FCC crystal with two different lattice constants: the experimental lattice constant $a_{\mathrm{e}}$, which equals $4.022 \AA$ when finite-temperature and zero-point effects are removed, ${ }^{19}$ and the lattice constant corresponding to the minimum-energy lattice constant of the PEF, which we will denote $a_{\mathrm{m}}$. The two energies are labeled $E_{\mathrm{b}}\left(a_{\mathrm{e}}\right)$ and $E_{\mathrm{b}}\left(a_{\mathrm{m}}\right)$, and their difference provides an indirect measure of the accuracy of the fitted lattice constant $a_{\mathrm{m}}$. The cohesive energy of a cluster is the atomization energy of the cluster divided by the number of atoms in the cluster. The bulk cohesive energy $E_{\mathrm{b}}$ for an arbitrary value of $a$ is approximated by computing the cohesive energies $Q_{\mathrm{N}}(a)$ for two FCC quasispherical clusters (QSCs) with $N=55$ and 321 and lattice constant $a$ and extrapolating to infinite $N$ by assuming a linear dependence on $N^{-1 / 3}$, i.e.,

$$
E_{\mathrm{b}}(a)=\frac{55^{-1 / 3} Q_{321}(a)-321^{-1 / 3} Q_{55}(a)}{55^{-1 / 3}-321^{-1 / 3}}
$$

We verified that the linear relationship in eq 1 is a good approximation for the analytic PEFs considered here and that extrapolating from the two QSC energies (for $\mathrm{Al}_{55}$ and $\mathrm{Al}_{321}$ ) provides a good estimate (for $N \geq 55$ ) of the value obtained by fitting to a larger series of QSC energies with various large values of $N$. The lattice constant $a_{\mathrm{m}}$ predicted by the PEF is obtained by finding the minimum of $E_{\mathrm{b}}(a)$ with respect to $a$.
Several mean unsigned errors (MUEs) were calculated using the test set as follows. Let $E_{k}^{i}$ stand for the PBE0/MG3 energy of geometry $i$ for cluster size $N_{\mathrm{k}}$, where $i=1, \ldots, n_{\mathrm{k}}$, i.e., $n_{\mathrm{k}}$ is the number of geometries included in the test set of cluster size $N_{\mathrm{k}}$. Similarly, the corresponding energy computed for one of the analytic PEFs is $G_{k}^{i}$. In each case we take the zero of energy as the energy of the infinitely separated atoms in the ground state. Thus, if the absolute energies from the PBE0/ MG3 and analytic PEF calculations are $e_{k}^{i}$ and $g_{k}^{i}$, respectively, we have

$$
E_{k}^{i} \equiv e_{k}^{i}-N_{k} e_{1}^{1}
$$

and

$$
G_{k}^{i} \equiv g_{k}^{i}-N_{k} g_{1}^{1}
$$

where $e_{1}^{1}$ and $g_{1}^{1}$ are atomic energies. The unsigned error for each geometry in the data set is

$$
\Delta E_{k}^{i}=\left|E_{k}^{i}-G_{k}^{i}\right|
$$

The unsigned error in the energy difference of any pair of geometries, $i$ and $i^{\prime}$, of a single cluster size $N_{\mathrm{k}}$ is

$$
\Delta \Delta E_{k}^{i i^{\prime}}=\left|\left(E_{k}^{i^{\prime}}-E_{k}^{i}\right)-\left(G_{k}^{i^{\prime}}-G_{k}^{i}\right)\right|
$$

and this error measures the accuracy of the shape of the PEF. The MUE per atom for each cluster size $N_{\mathrm{k}}$ is defined as

$$
\epsilon_{k}=\frac{1}{2}\left(\frac{2}{N_{k} n_{k}\left(n_{k}-1\right)} \sum_{i=1}^{n_{k}} \sum_{i^{\prime}>i}^{n_{k}} \Delta \Delta E_{k}^{i \prime^{\prime}}+\frac{1}{N_{k} n_{k}} \sum_{i=1}^{n_{k}} \Delta E_{k}^{i}\right)
$$

The average error for the five cluster sizes (i.e., the average of $\left.\epsilon_{k}, k=1-5\right)$ is labeled $\epsilon_{\mathrm{c}}$. The unsigned error in the bulk cohesive energy is given by

$$
\epsilon_{\mathrm{b}}=\frac{1}{2}\left(\left|E_{\mathrm{b}}\left(a_{\mathrm{m}}\right)-E_{\mathrm{b}}^{\mathrm{e}}\right|+\left|E_{\mathrm{b}}\left(a_{\mathrm{e}}\right)-E_{\mathrm{b}}^{\mathrm{e}}\right|\right)
$$

where $E_{\mathrm{b}}^{\mathrm{e}}$ is the experimental FCC bulk cohesive energy for aluminum $^{20,21}(3.43 \mathrm{eV})$ and excludes vibrational zero-point contributions as discussed elsewhere. ${ }^{11}$ The "total" MUE per atom $\epsilon$ is the weighted average of $\epsilon_{\mathrm{b}}$ and the five values of $\epsilon_{k}$ :

$$
\epsilon=\frac{1}{N_{\mathrm{av}}}\left(\epsilon_{\mathrm{b}}+\sum_{k=1}^{5} N_{k} \epsilon_{k}\right)
$$

where

$$
N_{\mathrm{av}}=\frac{1}{6}\left(1+\sum_{k=1}^{5} N_{k}\right)
$$

In eq 8 , the five per-atom MUEs for the clusters $\epsilon_{k}$ are weighted according to their cluster sizes, thus giving more relative weight to the $\mathrm{Al}_{13}$ data points than to the smaller clusters. The experimental bulk cohesive energy and lattice constant are included in the data set with a small relative weight to test whether the analytic PEFs are reasonable for clusters larger than $\mathrm{Al}_{13}$ and to ensure that the newly parametrized models extrapolate in a reasonable, even if not quantitative, way to the bulk limit, but it is not the goal of this paper to develop analytic PEFs that predict quantitative values for bulk properties. 
A microgenetic algorithm ${ }^{17,18}$ was used to optimize a set of parameters using eq 8 as the unfitness function. Note that to avoid a relatively expensive calculation, $E_{\mathrm{b}}\left(a_{\mathrm{m}}\right)$ was approximated during the reparametrization procedure by fitting $E_{\mathrm{b}}(a)$ with three different values of $a\left(1.1 a_{\mathrm{e}}, a_{\mathrm{e}}\right.$, and $\left.0.9 a_{\mathrm{e}}\right)$ to a parabola and setting $a_{\mathrm{m}}$ equal to the value of $a$ at the minimum of the parabola. This approximation was used only during the fitting procedure, and whenever errors are tabulated in the present paper, eq 1 is minimized numerically to obtain $a_{\mathrm{m}}$ and $E_{\mathrm{b}}\left(a_{\mathrm{m}}\right)$

Using a microgenetic algorithm does not guarantee that the best set of parameters for a particular error function (unfitness function) is found (because the optimization may converge to a local minimum), and often several sets of parameters may be found that give similar values for the unfitness function but have varying distributions of errors over the data set. Furthermore, the microgenetic algorithm may determine a "best" set of parameters that no longer has a physically meaningful interpretation. We have, however, attempted a thorough search of parameter space by optimizing several initial guesses for the parameters for each PEF, and in every case we continued the optimization until we found a physically meaningful set of parameters that gave the smallest value we could obtain for $\epsilon$. Therefore, the results allow for a discussion of the relative success of each type of PEF, which is a goal of the present paper.

\section{Potential Energy Functions}

In this section, nineteen PEFs for aluminum that have been collected from the literature are described and categorized. (Note that the term PEF is used to indicate a particular functional form and a particular choice of parameters.) We do not present an exhaustive review of the available PEFs (see refs 22 and 23 for recent reviews of PEFs parametrized for a variety of materials systems, including aluminum), but we have attempted to include $\mathrm{PEFs}$ featuring several different functional forms based on a variety of different theoretical justifications or empirical strategies. When PEFs are coded from the literature, it is always possible that a typographical error is present in the published work. We hope that we do not misrepresent the accuracy or inaccuracy of any PEF due to such an error, and we encourage authors to make their PEFs available in online databases such as POTLIB-online ${ }^{24}$ (where all of the PEFs mentioned in this article are available for download as Fortran routines). Several PEFs that have not been previously parametrized for aluminum are also presented and discussed.

III.A. Pairwise Additive PEFs. In the absence of external forces, the total energy of $N$ interacting atoms may be written as $^{25}$

$$
V=V_{1}+V_{2}+\ldots+V_{N}
$$

where $V_{\mathrm{N}}$ is the sum of $N$-body interaction energies; i.e., $V_{1}$ is the energy of $N$ infinitely separated atoms,

$$
\begin{gathered}
V_{2}=\sum_{\alpha>\beta} U_{2}\left(R_{\alpha \beta}\right) \\
V_{3}=\sum_{\alpha>\beta>\gamma} U_{3}\left(R_{\alpha \beta}, R_{\beta \gamma}, R_{\alpha \gamma}\right), \text { etc. } \\
R_{\alpha \beta}=\left|\mathbf{R}_{\alpha}-\mathbf{R}_{\beta}\right|
\end{gathered}
$$

and $\mathbf{R}_{\alpha}$ denotes the coordinates of atom $\alpha$. Note that the zero of energy is defined such that $V_{1}=0$ for all of the PEFs considered in the present work.

It is not clear how quickly the sum in eq 10 converges, especially for metals, which have long-range correlations. Nevertheless it is a popular approach to truncate eq 10, often at only two or a few terms. PEFs that truncate the expansion at $V_{2}$ are called pairwise additive (PA), and they are well-known to be inadequate for quantitative work. ${ }^{26-29}$ Four pairwiseadditive PEFs are considered in the present work, three of which have been previously parametrized for aluminum.

Halicioğlu and Pound ${ }^{30}$ (HalP) parametrized a Lennard-Jones PEF for cubic metals by fitting to the crystal properties of aluminum. The PEF may be written in the general form

$$
U_{2}\left(R_{\alpha \beta}\right)=A\left[\left(\frac{\sigma}{R_{\alpha \beta}}\right)^{m}-\left(\frac{\sigma}{R_{\alpha \beta}}\right)^{n}\right]
$$

where $\sigma=(n / m)^{1 /(m-n)} R_{\mathrm{e}}, R_{\mathrm{e}}$ denotes the minimum-energy bond distance of the two-body interaction, and $m>n$.

The Pettifor-Ward (PetW) potential has the form

$$
U_{2}\left(R_{\alpha \beta}\right)=\frac{2 Z^{2}}{R_{\alpha \beta}} \sum_{n=1}^{3} A_{n} \cos \left(k_{n} R_{\alpha \beta}+a_{n}\right) \mathrm{e}^{-\kappa_{n} R_{\alpha \beta}}
$$

and it was developed for simple metals and applied to study structural phase transitions. ${ }^{31}$ The parameters were obtained using second-order pseudopotential theory. ${ }^{32,33}$

Hase and co-workers (deSPH) used a Morse curve with a parametrized range parameter to model aluminum clusters. ${ }^{34}$ The PEF (which we will call deSPH/M) has the form

$$
U_{2}\left(R_{\alpha \beta}\right)=D_{\mathrm{e}}\left(\exp \left[-2 a Y_{\alpha \beta}\right]-2 \exp \left[-a Y_{\alpha \beta}\right]\right)
$$

where

$$
a=a_{0}+a_{2} Y_{\alpha \beta}^{2}+a_{3} Y_{\alpha \beta}^{3}
$$

and

$$
Y_{\alpha \beta}=R_{\alpha \beta}-R_{\mathrm{e}}
$$

The deSPH/M PEF was developed to model $\mathrm{Al}_{6}$ by fitting to the bond distances and frequencies for $\mathrm{Al}_{6}$ predicted by a Lennard-Jones/Axilrod-Teller analytic PEF (labeled deSPH/ LJAT and included in section III.D).

The extended Rydberg ${ }^{25,35}$ (ER) functional form is

$$
U_{2}\left(R_{\alpha \beta}\right)=-D_{\mathrm{e}}\left(1+a_{1} Y_{\alpha \beta}+a_{2} Y_{\alpha \beta}{ }^{2}+a_{3} Y_{\alpha \beta}{ }^{3}\right) \exp \left(-a_{1} Y_{\alpha \beta}\right)
$$

where $Y_{\alpha \beta}$ is given in eq 18. This PEF has not previously been parametrized for aluminum; it is introduced here because it is flexible enough to provide an accurate representation of $V_{2}$.

Parameters for the PA PEFs obtained from the literature are given in the first numerical column of Table 1; optimized values in the tables are explained in section IV.B.

III.B. Functional Forms for Explicit Many-Body Effects. One strategy for modeling many-body effects is to modify a two-body interaction in the presence of nearby atoms, i.e.,

$$
V=\sum_{\alpha>\beta} U_{2}\left(R_{\alpha \beta}\right) f_{\alpha \beta}^{\mathrm{MB}}
$$

where $f_{\alpha \beta}^{\mathrm{MB}}$ is a many-body function that is a function of all bond distances in the system involving either atom $\alpha$ or atom $\beta$; because $f_{\alpha \beta}^{\mathrm{MB}}$ is designed to model many-body effects, it 
TABLE 1: Parameters for the Pairwise-Additive PEFs

\begin{tabular}{clcc}
\hline PEF & parameter & literature value & optimized value \\
\hline HalP & $A(\mathrm{eV})$ & 1.569 & 0.2396 \\
& $R_{\mathrm{e}}(\AA)$ & 2.941 & 2.934 \\
& $m$ & 12.00 & 8.144 \\
PetW & $n$ & 6.000 & 5.804 \\
& $Z$ & 3.000 & 3.000 \\
& $A_{1}$ & 7.964 & 67.16 \\
& $A_{2}$ & 1.275 & 0.1641 \\
& $A_{3}$ & 0.03000 & 57.38 \\
& $a_{1} / \pi$ & -0.4410 & 15.13 \\
& $a_{2} / \pi$ & 0.8320 & 30.84 \\
& $a_{3} / \pi$ & 0.4310 & 23.90 \\
& $k_{1} / k_{2 \mathrm{~F}}$ & 0.1560 & 0.7146 \\
& $k_{2} / k_{2 \mathrm{~F}}$ & 0.6440 & 0.6642 \\
& $k_{3} / k_{2 \mathrm{~F}}$ & 0.9580 & 0.1479 \\
& $\kappa_{1} / k_{2 \mathrm{~F}}$ & 0.7930 & 1.889 \\
& $\kappa_{2} / k_{2 \mathrm{~F}}$ & 0.6980 & 0.2771 \\
& $\kappa_{3} / k_{2 \mathrm{~F}}$ & 0.2780 & 1.926 \\
$\operatorname{deSPH} / \mathrm{M}$ & $k_{2 \mathrm{~F}}\left(\AA^{-1}\right)$ & 3.504 & 3.504 \\
& $D_{\mathrm{e}}(\mathrm{eV})$ & 0.5608 & 0.7989 \\
& $R_{\mathrm{e}}(\AA)$ & 2.834 & 3.183 \\
& $a_{0}\left(\AA^{-1}\right)$ & 0.5282 & 0.5457 \\
& $a_{2}\left(\AA^{-3}\right)$ & 0.004390 & 0.2995 \\
& $a_{3}\left(\AA^{-4}\right)$ & 5.109 & 1.749
\end{tabular}

equals unity when atoms $\alpha$ and $\beta$ are close to each other and are far from all other atoms. As an alternate to eq 20, one may write

$$
V=\sum_{\alpha>\beta} U_{2}\left(R_{\alpha \beta}\right)-\sum_{\alpha>\beta} U_{2}^{\prime}\left(R_{\alpha \beta}\right)\left(1-f_{\alpha \beta}^{\mathrm{MB}}\right)
$$

where $U_{2}^{\prime}\left(R_{\alpha \beta}\right)$ need not be the same as $U_{2}\left(R_{\alpha \beta}\right)$. Obviously, eq 20 is simpler, but eq 21 provides additional flexibility, and both strategies are pursued.

We consider three functional forms for the many-body function; they are designed to model screening, bond-angle, and coordination number effects, respectively. The screening function, developed by Ho and co-workers, ${ }^{36-39}$ has the form

$$
f_{\alpha \beta}^{\mathrm{S}}=1-\tanh \left(\chi_{\alpha \beta}^{\mathrm{S}}\right)
$$

where

$$
\chi_{\alpha \beta}^{\mathrm{S}}=\kappa_{1} \sum_{\gamma \neq \alpha, \beta} \exp \left[-\kappa_{2}\left(R_{\alpha \gamma}+R_{\beta \gamma}\right)^{\kappa_{3}} / R_{\alpha \beta}{ }^{\kappa_{3}}\right]
$$

Next, we consider two new many-body functions developed specifically for the present work. First is a bond angle (BA) function capable of modeling three- and four-body effects:

$$
\begin{gathered}
f_{\alpha \beta}^{\mathrm{BA}}=1-\tanh \left(\chi_{\alpha \beta}^{\mathrm{BA}}\right) \\
\chi_{\alpha \beta}^{\mathrm{BA}}=\kappa_{1} \sum_{\gamma \neq \alpha, \beta \delta \neq \alpha, \beta} \sum_{\gamma \neq \alpha} \exp \left[-\kappa_{2}\left(R_{\alpha \gamma}^{\kappa_{3}}+R_{\delta \beta}^{\kappa_{3}}\right)\right]
\end{gathered}
$$

The screening and bond-angle functions are closely related, and both model the physical effect of weakening the interaction of two atoms in the presence of other atoms. Note that the screening function in eq 22 depends on the bond distance $R_{\alpha \beta}$ of the interaction being weakened, whereas the bond angle function in eq 24 does not.

Finally, we consider the effect of coordination number. The effective coordination number of atom $\alpha$ is defined as

$$
g_{\alpha}=\sum_{\alpha^{\prime} \neq \alpha} f_{g}\left(R_{\alpha \alpha^{\prime}}\right)
$$

where $g_{\alpha}$ depends on all of the internuclear distances involving atom $\alpha$, and

$$
f_{g}(R)= \begin{cases}\exp \left(\gamma_{1}+\frac{\gamma_{1} \gamma_{2}}{R-\gamma_{2}}\right) & \text { if } R<\gamma^{2} \\ 0 & \text { if } R \geq \gamma_{2}\end{cases}
$$

i.e., eq 26 counts the number of atoms near atom $\alpha$, weighted by their distance from atom $\alpha$ using the weighting function in eq 27. The functional form for the coordination number $(\mathrm{CN})$ term is

$$
f^{\mathrm{CN}}=1-d\left(1-G_{\alpha \beta}\right)
$$

where

$$
G_{\alpha \beta}=\frac{1}{1+\left(\frac{g_{\alpha}-f_{g}\left(R_{\alpha \beta}\right)}{g_{0}}\right)^{\gamma}} \frac{1}{1+\left(\frac{g_{\beta}-f_{g}\left(R_{\alpha \beta}\right)}{g_{0}}\right)^{\gamma}}
$$

and $d, \gamma$, and $g_{0}$ are adjustable parameters. It is convenient to think of $g_{0}$ as a reference coordination number. Equation 29 always has a value between zero and unity, so that the effect of eq 28 is to weaken the $\alpha-\beta$ bond as the number of nearby neighbors increases.

The screening function has not been previously parametrized for aluminum, and the bond angle and coordination number functions are newly presented in this work. All three functional forms may be implemented using eq 20, where they will be labeled $\mathrm{S}, \mathrm{BA}$, and $\mathrm{CN}$, respectively, or using the extended (E) form in eq 21, where they will be labeled ES, EBA, and ECN, respectively.

III.C. Embedded-Atom (EA) Methods. Another approach to developing analytic PEFs that is widely applied to bulk systems incorporates many-body effects by considering the energy required to place an impurity (an atom) into a lattice. ${ }^{40}$ In this approach the energetic effects of this placement are represented by an embedding function $F_{\alpha}$, which is a function of the local electron density. PEFs based on this approach have the general form

$$
V=\sum_{\alpha>\beta} U_{2}\left(R_{\alpha \beta}\right)+\sum_{\alpha} F_{\alpha}
$$

where $U_{2}$ is an effective two-body interaction, and $F_{\alpha}$ is an energy functional that, in general, depends on the local electron density at atom $\alpha$ and therefore on the geometry of the system. Several applications of eq 30 have appeared which vary in their derivations and prescriptions for $U_{2}$ and $F_{\alpha}$. Very often the embedding function $F_{\alpha}$ is written as the square root of a densitylike quantity such as a sum of exponentials. We refer to PEFs based on eq 30 as embedded-atom-type, or for simplicity, as embedded atom (EA) methods, and this category includes the "glue", ${ }^{41,42}$ Finnis - Sinclair, ${ }^{43}$ second-moment approximation to tight binding, ${ }^{44,45}$ Sutton-Chen, ${ }^{46}$ and several other related ${ }^{47-49}$ methods. Nine EA PEFs that have been previously parametrized for aluminum are considered in the present work. In addition, one PEF based on effective medium theory ${ }^{50}$ (EMT), which is similar to the EA approach, is also included. (Note that for convenience the EMT PEF is grouped with the EA PEFs.)

The Gollisch ${ }^{51}$ (Gol) PEF was used to study molecular, cluster, and surface properties and uses an exponential embedding function. It has

$$
U_{2}\left(R_{\alpha \beta}\right)=A \exp \left(-\alpha R_{\alpha \beta}\right)
$$


and

$$
F_{\alpha}=-B\left(\sum_{\beta \neq \alpha} \exp \left(-b R_{\alpha \beta}\right)\right)^{n}
$$

The parameters for the Gol PEF were determined from the bulk properties of aluminum.

The PEF of Betz and Husinsky (BetH) was used to simulate collisions of aluminum clusters with a copper surface. ${ }^{52}$ The functional form is mathematically identical to eqs 31 and 32 with $n=1 / 2$. The parameters for the BetH PEF were determined by fitting to the lattice constant, cohesive energy, bulk modulus, and average shear modulus of aluminum.

Three PEFs based on the second-moment approximation to tight binding 44,45 (TB- $\mu_{2}$ ) are included. The TB- $\mu_{2}$ functional form is usually applied with

$$
U_{2}\left(R_{\alpha \beta}\right)=A \exp \left[-p\left(R_{\alpha \beta} / R_{\mathrm{e}}-1\right)\right]
$$

and

$$
F_{\alpha}=-B\left(\sum_{\beta \neq \alpha} \exp \left[-2 q\left(R_{\alpha \beta} / R_{\mathrm{e}}-1\right)\right]\right)^{1 / 2}
$$

We note that eqs 33 and 34 are mathematically equivalent to eqs 31 and 32 with $n=1 / 2$. The Cleri-Rosato ${ }^{53}$ (CleR) PEF was applied to point-defect properties, lattice dynamics, and finite temperature behavior of metals and alloys, and its parameters were determined ${ }^{53}$ from the experimental values of the cohesive energy, lattice parameter, and elastic constants of aluminum. The Al-only limits of two TB- $\mu_{2}$ PEFs developed by Papaconstantopoulos and co-workers (PapCEP and PapKEP $)^{54,55}$ that were developed to model $\mathrm{Al}-\mathrm{Ni}$ alloys are also tested. These PEFs were developed ${ }^{54,55}$ by fitting to ab initio calculations of the bulk structure.

The Sutton-Chen ${ }^{46}$ (SutC) PEF uses

$$
U_{2}\left(R_{\alpha \beta}\right)=A\left(\frac{a}{R_{\alpha \beta}}\right)^{m}
$$

and terms involving $R_{\alpha \beta}^{-m}$ in the embedding function

$$
F_{\alpha}=-B\left(\sum_{\beta \neq \alpha}\left(\frac{a}{R_{\alpha \beta}}\right)^{n}\right)^{1 / 2}
$$

Parameters for the SutC PEF were determined ${ }^{46}$ by fitting to the crystal structure, cohesive energy, bulk modulus, and elastic constants of aluminum.

Mei and Davenport (MeiD) developed parameters for aluminum ${ }^{56}$ for a modified embedded atom PEF. ${ }^{57}$ The parameters were determined to reproduce the experimental cohesive energy, lattice constant, vacancy-formation energy, and elastic constants at $0 \mathrm{~K}$. The functional form is given by

$$
\begin{aligned}
& \begin{array}{l}
U_{2}\left(R_{\alpha \beta}\right)=\phi_{0}\left[1-d\left(R_{\alpha \beta} / R_{0}-1\right)\right] \times \\
\quad \exp \left[-c\left(R_{\alpha \beta} / R_{0}-1\right)\right] \Delta_{\mathrm{CO}}\left(R_{\alpha \beta}\right)
\end{array} \\
& F_{\alpha}=-E_{c}\left[1-(a / b) \ln \rho_{\alpha}\right] \rho_{\alpha}^{a / b}+{ }^{1}{ }_{2} \phi_{0} \sum_{m=1}^{3} s_{\mathrm{m}} \times \\
& \exp [-(\sqrt{m}-1) c]\left[1+(\sqrt{m}-1) d-\sqrt{m}(d / b) \ln \rho_{\alpha}\right] \rho_{\alpha}{ }^{m^{1 / 2} c / b}
\end{aligned}
$$

$$
\begin{gathered}
\rho_{\alpha}=\sum_{\beta \neq \alpha} \Delta\left(R_{\alpha \beta}\right) \sum_{l=0}^{5}\left(C_{l} / 12\right)\left(R_{0} / R_{\alpha \beta}\right)^{l} \\
\Delta_{\mathrm{CO}}(R)= \begin{cases}1 & R \leq R_{n} \\
(1-x)^{3}\left(1+3 x+6 x^{2}\right) & R_{n}<R<R_{c} \\
0 & R \geq R_{c}\end{cases}
\end{gathered}
$$

where

$$
x=\left(R-R_{n}\right) /\left(R_{c}-R_{n}\right)
$$

and $R_{\mathrm{n}}$ and $R_{\mathrm{c}}$ equal $1.75 R_{0}$ and $1.95 R_{0}$, respectively.

Streitz and Mintmire (StrM) developed a PEF for use in modeling the oxidation of bulk aluminum, which was fitted to experimental data for the FCC crystal. ${ }^{58}$ The metal-only part of the PEF is included in the present work. This PEF uses

$$
\begin{aligned}
U_{2}\left(R_{\alpha \beta}\right)= & 2 A_{1} \exp \left[-a_{1}\left(R_{\alpha \beta}-R_{\mathrm{e}}\right) / 2\right]- \\
& A_{2}\left[1+a_{2}\left(R_{\alpha \beta}-R_{\mathrm{e}}\right)\right] \exp \left[-a_{2}\left(R_{\alpha \beta}-R_{\mathrm{e}}\right)\right]
\end{aligned}
$$

and an embedding function equivalent to those given in eqs 32 and 34

$$
F_{\alpha}=-B\left(\sum_{\beta \neq \alpha} \exp \left[-a_{1}\left(R_{\alpha \beta}-R_{\mathrm{e}}\right)\right]\right)^{1 / 2}
$$

Mishin, Farkas, Mehl, and Papaconstantopoulos (MisFMP) developed and fit a $\mathrm{PEF}^{59}$ based on experimental and ab initio calculations of the bulk metal. The PEF has

$$
\begin{aligned}
& U_{2}\left(R_{\alpha \beta}\right)=\left[A_{1} M\left(R_{\alpha \beta}, R_{1}, a_{1}\right)+\right. \\
& \left.A_{2} M\left(R_{\alpha \beta}, R_{2}, a_{2}\right)+A_{3}\right] \psi\left(\frac{R_{\alpha \beta}-R_{c}}{h}\right)
\end{aligned}
$$

and

$$
F_{\alpha}=F_{0}+\frac{1}{2} F_{2}\left(\bar{\rho}_{\alpha}-1\right)+x_{\alpha}
$$

where

$$
\begin{gathered}
x_{\alpha}=\sum_{i=1}^{3} q_{i}\left(\bar{\rho}_{\alpha}-1\right)^{i+2} \\
\bar{\rho}_{\alpha}=\sum_{\beta \neq \alpha}\left[c \exp \left(-b_{1}\left(R_{\alpha \beta}-R_{3}\right)^{2}\right)+\right. \\
\left.\exp \left(-b_{2}\left(R_{\alpha \beta}-R_{4}\right)\right)\right] \psi\left(\frac{R_{\alpha \beta}-R_{c}}{h}\right) \\
M\left(R_{\alpha \beta}, R_{i}, a_{i}\right)=\exp \left[-2 a_{i}\left(R_{\alpha \beta}-R_{i}\right)\right]- \\
2 \exp \left[-a_{i}\left(R_{\alpha \beta}-R_{i}\right)\right]
\end{gathered}
$$

and

$$
\psi(x)= \begin{cases}0 & \text { for } x \geq 0 \\ x^{4} /\left(1+x^{4}\right) & \text { for } x<0\end{cases}
$$

The Jacobsen (Jac) $\mathrm{PEF}^{60}$ is based on EMT and may be written in the form of eq 30 , but it less awkward to write it as follows:

$$
V=\sum_{\alpha} E_{c}\left(\bar{n}_{\alpha}\right)+E_{A S}
$$


TABLE 2: Parameters for the Embedded Atom PEFs

\begin{tabular}{|c|c|c|c|c|c|c|c|}
\hline PEF & parameter & literature value & optimized value & PEF & parameter & literature value & optimized value \\
\hline \multirow[t]{5}{*}{ Gol } & $A(\mathrm{eV})$ & 350.4 & 641.3 & MeiD (cont'd) & $C_{3}$ & -47.16 & -43.63 \\
\hline & $B(\mathrm{eV})$ & 10.38 & 12.22 & & $C_{4}$ & 36.19 & 28.12 \\
\hline & $a\left(\AA^{-1}\right)$ & 2.734 & 2.991 & & $C_{5}$ & -8.608 & -6.751 \\
\hline & $b\left(\AA^{-1}\right)$ & 1.368 & 1.364 & & $s_{1}$ & 12.00 & 8.658 \\
\hline & $n$ & 0.6000 & 0.6626 & & $s_{2}$ & 6.000 & 4.170 \\
\hline \multirow[t]{5}{*}{ BetH } & $A(\mathrm{eV})$ & 1220 & 2450 & & $s_{3}$ & 24.00 & 27.91 \\
\hline & $B(\mathrm{eV})$ & 13.25 & 7.085 & StrM & $A_{1}(\mathrm{eV})$ & 0.07502 & 0.08510 \\
\hline & $a\left(\AA^{-1}\right)$ & 3.004 & 3.454 & & $A_{2}(\mathrm{eV})$ & 0.1595 & 0.4101 \\
\hline & $b\left(\AA^{-1}\right)$ & 1.637 & 1.326 & & $B(\mathrm{eV})$ & 0.7639 & 0.6871 \\
\hline & $n$ & 0.5000 & 0.5000 & & $a_{1}\left(\AA^{-1}\right)$ & 2.018 & 1.240 \\
\hline \multirow[t]{5}{*}{$\mathrm{CleR}^{a}$} & $A(\mathrm{eV})$ & 0.1221 & & & $a_{2}\left(\AA^{-1}\right)$ & 1.767 & 2.026 \\
\hline & $B(\mathrm{eV})$ & 1.316 & & & $R_{\mathrm{e}}(\AA)$ & 3.366 & 2.852 \\
\hline & $R_{\mathrm{e}}(\AA)$ & 2.864 & & MisFMP & $A_{1}(\mathrm{eV})$ & 2.652 & 1.389 \\
\hline & $p$ & 8.612 & & & $A_{2}(\mathrm{eV})$ & 0.007672 & 0.001762 \\
\hline & $q$ & 2.516 & & & $A_{3}(\mathrm{eV})$ & 1.030 & 0.1095 \\
\hline \multirow[t]{5}{*}{$\operatorname{PapCEP}^{a}$} & $A(\mathrm{eV})$ & 0.05500 & & & $R_{\mathrm{c}}(\AA)$ & 6.780 & 6.117 \\
\hline & $B(\mathrm{eV})$ & 0.9564 & & & $h(\AA)$ & 1.416 & 4.788 \\
\hline & $R_{\mathrm{e}}(\AA)$ & 2.831 & & & $a_{1}\left(\AA^{-1}\right)$ & 2.090 & 1.017 \\
\hline & $p$ & 10.90 & & & $a_{2}\left(\AA^{-1}\right)$ & 1.030 & 1.313 \\
\hline & $q$ & 1.513 & & & $b_{1}\left(\AA^{-2}\right)$ & 1.921 & 0.5742 \\
\hline \multirow[t]{5}{*}{ PapKEP $^{a}$} & $A(\mathrm{eV})$ & 0.03340 & & & $b_{2}\left(\AA^{-1}\right)$ & 0.4259 & 0.2615 \\
\hline & $B(\mathrm{eV})$ & 0.7981 & & & $c$ & 0.05475 & 0.09980 \\
\hline & $R_{\mathrm{e}}(\AA)$ & 2.812 & & & $R_{1}(\AA)$ & 1.069 & 2.340 \\
\hline & $p$ & 14.61 & & & $R_{2}(\AA)$ & 6.458 & 5.139 \\
\hline & $q$ & 1.112 & & & $R_{3}(\AA)$ & 2.747 & 4.411 \\
\hline \multirow[t]{5}{*}{ SutC } & $A(\mathrm{eV})$ & 0.03315 & 0.02304 & & $R_{4}(\AA)$ & -6.926 & -6.123 \\
\hline & $B(\mathrm{eV})$ & 0.5436 & 0.5649 & & $F_{0}(\mathrm{eV})$ & -2.815 & -3.139 \\
\hline & $a(\AA)$ & 4.050 & 4.050 & & $F_{2}(\mathrm{eV})$ & 5.577 & 4.718 \\
\hline & $m$ & 7.000 & 6.853 & & $q_{1}(\mathrm{eV})$ & -6.247 & -3.625 \\
\hline & $n$ & 6.000 & 4.2056 & & $q_{2}(\mathrm{eV})$ & -21.53 & -23.15 \\
\hline \multirow[t]{10}{*}{ MeiD } & $\phi_{0}(\mathrm{eV})$ & 0.1318 & 0.3958 & & $q_{3}(\mathrm{eV})$ & -15.30 & -20.11 \\
\hline & $R_{0}(\AA)$ & 2.864 & 2.625 & $\mathrm{Jac}$ & $E_{0}(\mathrm{eV})$ & -3.280 & -3.446 \\
\hline & $E_{\mathrm{c}}(\mathrm{eV})$ & 3.390 & 3.649 & & $E_{2}(\mathrm{eV})$ & 1.120 & 2.345 \\
\hline & $a$ & 4.600 & 5.194 & & $E_{3}(\mathrm{eV})$ & -0.3500 & -0.7848 \\
\hline & $b$ & 7.100 & 4.749 & & $n_{0}\left(\AA^{-3}\right)$ & 0.04724 & 0.03536 \\
\hline & $c$ & 7.348 & 5.754 & & $\eta\left(\AA^{-1}\right)$ & 3.780 & 2.657 \\
\hline & $d$ & 7.350 & 7.664 & & $\eta_{1}\left(\AA^{-1}\right)$ & 0.5669 & 0.7111 \\
\hline & $C_{0}$ & 0.6409 & 0.01057 & & $s_{0}(\AA)$ & 1.588 & 1.879 \\
\hline & $C_{1}$ & -6.838 & -7.999 & & $a\left(\mathrm{eV}^{-3}\right)$ & 189.7 & 262.2 \\
\hline & $C_{2}$ & 26.76 & 31.69 & & $b$ & 1.810 & 1.566 \\
\hline
\end{tabular}

${ }^{a}$ The CleR, PapCEP, and PapKEP PEFs have functional forms that are mathematically equivalent the BetH PEF and were not individually reparametrized.

where

$$
\begin{gathered}
E_{c}\left(\bar{n}_{\alpha}\right)=E_{0}+E_{2}\left(\frac{\bar{n}_{\alpha}}{n_{0}}-1\right)^{2}+E_{3}\left(\frac{\bar{n}_{\alpha}}{n_{0}}-1\right)^{3} \\
E_{\mathrm{AS}}-a n_{0} \sum_{\alpha}\left(E_{\mathrm{AS}, \alpha}^{(1)}-E_{\mathrm{AS}, \alpha}^{(2)}\right) \\
E_{\mathrm{AS}, \alpha}^{(1)}=\left[\frac{1}{12} \sum_{\beta \neq \alpha} \exp \left[-\eta_{2}\left(R_{\alpha \beta} / b s_{0}\right)\right]\right]^{\eta /\left(\eta+\eta_{1}\right)} \\
E_{\mathrm{AS}, \alpha}^{(1)}=\left[\frac{1}{12} \sum_{\beta \neq \alpha} \exp \left[-\eta\left(R_{\alpha \beta} / b-s_{0}\right)\right]\right] \\
\bar{n}_{\alpha}=n_{0} E_{\mathrm{AS}, \alpha}^{(1)} \\
\eta_{2}=\left(\eta+\eta_{1}\right) / b
\end{gathered}
$$

The parameters were determined ${ }^{60}$ with the help of local density functional calculations. This PEF was used to study the cohesive properties of metals.

Parameters for the EA PEFs obtained from the literature are given in Table 2 .
III.D. Nonpairwise Additive Potentials with Two- and Three-Body Terms. Numerous other three-body terms have been developed to model various physical effects, and several PEFs that include such terms are considered in the present work. We denote PEFs based on eq 10 and truncated at $V_{3}$ as nonpairwise additive (NPA) PEFs. Of course, the PEFs of sections III.B and III.C can also be called nonpairwise, but we will use NPA to refer to the PEFs discussed here because they explicitly add two-body and three-body effects.

Erkoç proposed three PEFs for aluminum that are included in the present paper. The first two PEFs (which he labeled IV and $\mathrm{V}$ and which we will call ErkIV and ErkV) have the form $^{61-63}$

$$
\begin{gathered}
U_{2}\left(R_{\alpha \beta}\right)=A\left[\left(\frac{R_{\mathrm{e}}}{R_{\alpha \beta}}\right)^{2 n} \mathrm{e}^{-2 a\left(R_{\alpha \beta} / R_{\mathrm{e}}\right)^{2}}-\left(\frac{R_{\mathrm{e}}}{R_{\alpha \beta}}\right)^{n} \mathrm{e}^{-a\left(R_{\alpha \beta} / R_{\mathrm{e}}\right)^{2}}\right] \\
U_{3}\left(R_{\alpha \beta}, R_{\beta \gamma}, R_{\alpha \gamma}\right)= \\
B\left(U_{2}\left(R_{\alpha \beta}\right) f_{\alpha \beta \gamma}+U_{2}\left(R_{\alpha \gamma}\right) f_{\alpha \beta \gamma}+U_{2}\left(R_{\beta \gamma}\right) f_{\beta \gamma \alpha}\right)
\end{gathered}
$$

where $f_{\alpha \beta \gamma}$ is a heuristic three-body term given by

$$
F_{\alpha \beta \gamma}=\exp \left[-\left(R_{\alpha \gamma}{ }^{2}+R_{\beta \gamma}{ }^{2}\right) / R_{\mathrm{e}}{ }^{2}\right]
$$

Parameters for the ErkIV $\mathrm{PEF}^{61,62}$ were obtained by fitting $U_{2}$ 
to diatomic data, and then using the remaining parameter in $U_{3}$ to obtain the correct cohesive energy. The ErkV PEF ${ }^{63}$ differs from the ErkIV PEF only in the values of $A$ and $B$, which were reparametrized using a bulk stability condition.

The Axilrod-Teller (AT) triple-dipole term ${ }^{64}$ was designed to model long-range dispersion interactions and has been shown to be important for the quantitative modeling of rare gases and inert condensed phase materials ${ }^{26,29}$ including interactions with carbon nanotubes. ${ }^{28}$ Three PEFs that include the AT term are considered. Erkoç developed ${ }^{65}$ a PEF called VIII (which we call ErkVIII) for FCC systems and parametrized it to experimental bulk quantities:

$$
U_{2}\left(R_{\alpha \beta}\right)=A_{1}\left(\frac{R_{\mathrm{e}}}{R_{\alpha \beta}}\right)^{m}-A_{2}\left(\frac{R_{\mathrm{e}}}{R_{\alpha \beta}}\right)^{n}
$$

$$
\begin{aligned}
& U_{3}\left(R_{\alpha \beta}, R_{\beta \gamma}, R_{\alpha \gamma}\right)= \\
& \quad B_{1} G_{\alpha \beta \gamma}\left(R_{\alpha \beta}, R_{\beta \gamma}, R_{\alpha \gamma}\right)+B_{2} H_{\alpha \beta \gamma}\left(R_{\alpha \beta}, R_{\beta \gamma}, R_{\alpha \gamma}\right)
\end{aligned}
$$

where

$$
G_{\alpha \beta \gamma}=\frac{1+3 \cos \vartheta_{\alpha} \cos \vartheta_{\beta} \cos \vartheta_{\gamma}}{\left(R_{\alpha \beta} R_{\beta \gamma} R_{\alpha \gamma}\right)^{3}}
$$

is the Axilrod-Teller triple-dipole term, ${ }^{64}$

$$
\begin{aligned}
& H_{\alpha \beta \gamma}= \\
& \quad \frac{8 \cos \vartheta_{\gamma}-25 \cos 3 \vartheta_{\gamma}+6 \cos \left(\vartheta_{\alpha}-\vartheta_{\beta}\right)\left(3+5 \cos 2 \vartheta_{\gamma}\right)}{R_{\alpha \beta}{ }^{3} R_{\alpha \gamma}{ }^{4} R_{\beta \gamma}{ }^{4}}
\end{aligned}
$$

is the next leading term in the triple-dipole expansion, and $\vartheta_{\alpha}$ is the $\beta-\alpha-\gamma$ bond angle (similarly $\vartheta_{\beta}$ and $\vartheta_{\gamma}$ are the $\alpha-\beta-\gamma$ and $\alpha-\gamma-\beta$ bond angles).

The next two PEFs that are considered have the same functional form but have different sets of parameters. The PEFs of Pearson et al. ${ }^{66}$ (PeaTHT) and Hase and co-workers ${ }^{34}$ (deSPH/ LJAT) both use the Lennard-Jones two-body interaction in eq 14 and the Axilrod-Teller three-body term

$$
U_{3}\left(R_{\alpha \beta}, R_{\beta \gamma}, R_{\alpha \gamma}\right)=B G_{\alpha \beta \gamma}
$$

where $G_{\alpha \beta \gamma}$ is given in eq 62. The parameters for the PeaTHT PEF were obtained by fitting to the equilibrium bond lengths of the dimer and trimer and the lattice parameter and cohesive energy of bulk aluminum. The deSPH/LJAT PEF parameters were obtained by fitting to ab initio calculations for $\mathrm{Al}_{N}(N=$ $2-6,13)$ clusters.

A PEF was developed by Cox, Johnston, and Murrell ${ }^{67,68}$ (CoxJM) for cubic solids and was parametrized to reproduce the cohesive energy and bulk lattice spacing. The two-body term is a Rydberg function of the form

$$
U_{2}\left(R_{\alpha \beta}\right)=-D_{\mathrm{e}}\left(1+a_{2} \rho_{\alpha \beta}\right) \exp \left(-a_{2} \rho_{\alpha \beta}\right)
$$

where

$$
\rho_{\alpha \beta}=\frac{R_{\alpha \beta}-R_{\mathrm{e}}}{R_{\mathrm{e}}}
$$

and the three-body term has the form

$$
\begin{aligned}
& U_{3}\left(R_{\alpha \beta}, R_{\beta \gamma}, R_{\gamma \alpha}\right)=D_{\mathrm{e}}\left[c_{0}+c_{1} Q_{1}+c_{2} Q_{1}{ }^{2}+c_{3}\left(Q_{2}{ }^{2}-\right.\right. \\
& \left.\left.Q_{3}{ }^{2}\right)+c^{4} Q_{1}{ }^{3}+c_{5} Q_{1}\left(Q_{2}{ }^{2}-Q_{3}{ }^{2}\right)+c_{6}\left(Q_{3}{ }^{3}-3 Q_{3} Q_{2}{ }^{2}\right)\right] F
\end{aligned}
$$

where the generalized coordinates $Q_{\mathrm{i}}$ are defined by the transformation

$$
\left(\begin{array}{l}
Q_{1} \\
Q_{2} \\
Q_{3}
\end{array}\right)=\left(\begin{array}{lll}
\sqrt{1 / 3} & \sqrt{1 / 3} & \sqrt{1 / 3} \\
0 & \sqrt{1 / 2} & \sqrt{1 / 2} \\
\sqrt{2 / 3} & -\sqrt{1 / 6} & -\sqrt{1 / 6}
\end{array}\right)\left(\begin{array}{l}
\rho_{\alpha \beta} \\
\rho_{\beta \gamma} \\
\rho_{\gamma \alpha}
\end{array}\right)
$$

and the damping function is

$$
F=\operatorname{sech}\left(a_{3} Q_{1}\right)
$$

Parameters for the NPA PEFs obtained from the literature are given in Table 3.

No PEFs based on eq 10 that explicitly include four-body or higher terms in the expansion were found in the literature for aluminum, and none are considered in the present work.

III.E. Linear Scaling. The computer cost associated with evaluating the PEFs discussed in sections III.A and III.C scales as fast as $N^{2}$ for the PA and EA PEFs, and the cost scales as $N^{3}$ for the NPA and many-body PEFs in sections III.B and III.D, where $N$ is the number of atoms in the system. Physically, one expects that any single atom in a large system interacts significantly only with nearby atoms and that there is some cutoff distance at which the interaction between two atoms may be set to zero, i.e., the number of significant interactions scales as $N$. This linear scaling may be introduced smoothly into an analytic PEF using the cutoff function of Stillinger and Weber ${ }^{69}$ $f_{\mathrm{CO}}$, which is given by the formula

$$
f_{\mathrm{CO}}(R)= \begin{cases}\exp \left(\Delta+\frac{\Delta R_{\mathrm{CO}}}{R-R_{\mathrm{CO}}}\right) & \text { if } R<R_{\mathrm{CO}} \\ 0 & \text { if } R \geq R_{\mathrm{CO}}\end{cases}
$$

where $\Delta$ is a positive adjustable parameter, and $R_{\mathrm{CO}}$ is the distance at which the cutoff goes exactly to zero. Note this function has an infinite number of continuous derivatives for all values of $R$.

The cutoff function may be applied to two-body terms

$$
U_{2}^{\mathrm{CO}}=U_{2}\left(R_{\alpha \beta}\right) f_{\mathrm{CO}}\left(R_{\alpha \beta}\right)
$$

to three-body terms

$$
U_{3}^{\mathrm{CO}}=U_{3}\left(R_{\alpha \beta}, R_{\beta \gamma}, R_{\alpha \gamma}\right) f_{\mathrm{CO}}\left(R_{\alpha \beta}\right) f_{\mathrm{CO}}\left(R_{\beta \gamma}\right) f_{\mathrm{CO}}\left(R_{\alpha \gamma}\right)
$$

and to the EA embedding functions; e.g., eq 32 may be rewritten

$$
F_{\alpha}=-B\left(\sum_{\beta \neq \alpha} \exp \left(-b R_{\alpha \beta}\right) f_{\mathrm{CO}}\left(R_{\alpha \beta}\right)\right)^{n}
$$

When all of the terms are cut off in this way, the computational cost of the algorithm scales linearly with $N$ for large $N$.

The cutoff functions in eqs $70-73$ are incorporated in the PEFs presented in section IV.C but not in those discussed in sections IV.A and IV.B.

\section{Results and Discussion}

IV.A. Literature PEFs. The MUEs defined in eqs 6-8 were evaluated for all nineteen literature PEFs discussed in sections 
TABLE 3: Parameters for the PEFs with Two- and Three-Body Terms

\begin{tabular}{|c|c|c|c|}
\hline PEF & parameter & literature value & optimized value \\
\hline \multirow[t]{5}{*}{ ErkIV } & $A(\mathrm{eV})$ & 6.200 & 6.117 \\
\hline & $B$ & -1.554 & -1.589 \\
\hline & $R_{\mathrm{e}}(\AA)$ & 2.470 & 2.410 \\
\hline & $a$ & 0.6931 & 0.6931 \\
\hline & $n$ & 2.073 & 2.396 \\
\hline \multirow[t]{5}{*}{ ErkV $^{a}$} & $A(\mathrm{eV})$ & 1.634 & \\
\hline & $B$ & -0.6703 & \\
\hline & $R_{\mathrm{e}}(\AA)$ & 2.470 & \\
\hline & $a$ & 0.6931 & \\
\hline & $n$ & 2.073 & \\
\hline \multirow[t]{7}{*}{ ErkVIII } & $A_{1}(\mathrm{eV})$ & 1.860 & 1.491 \\
\hline & $A_{2}(\mathrm{eV})$ & 3.410 & 3.114 \\
\hline & $B_{1}\left(\mathrm{eV}^{9}\right)$ & 1643 & 2522 \\
\hline & $B_{2}\left(\mathrm{eV} \AA^{11}\right)$ & 1921 & 86.90 \\
\hline & $R_{\mathrm{e}}(\AA)$ & 2.510 & 2.510 \\
\hline & $m$ & 11.00 & 6.904 \\
\hline & $n$ & 6.000 & 4.843 \\
\hline \multirow{5}{*}{ PeaTHT } & $A(\mathrm{eV})$ & 4.864 & 122.4 \\
\hline & $R_{\mathrm{e}}(\AA)$ & 2.520 & 2.408 \\
\hline & $n$ & 6.000 & 4.949 \\
\hline & $m$ & 12.00 & 5.052 \\
\hline & $B\left(\mathrm{eV} \AA^{9}\right)$ & 2241 & 203.4 \\
\hline \multirow[t]{5}{*}{$\operatorname{deSPH} / \mathrm{LJAT}^{b}$} & $A(\mathrm{eV})$ & 4.600 & \\
\hline & $R_{\mathrm{e}}(\AA)$ & 2.635 & \\
\hline & $n$ & 6.000 & \\
\hline & $m$ & 12.00 & \\
\hline & $B\left(\mathrm{eV} \AA^{9}\right)$ & 3525 & \\
\hline \multirow[t]{11}{*}{ CoxJM } & $D_{\mathrm{e}}(\mathrm{eV})$ & 0.9073 & 1.250 \\
\hline & $R_{\mathrm{e}}(\AA)$ & 2.757 & 2.617 \\
\hline & $a_{2}$ & 7.000 & 4.751 \\
\hline & $a_{3}$ & 8.000 & 7.739 \\
\hline & $c_{0}$ & 0.2525 & 0.1822 \\
\hline & $c_{1}$ & -0.4671 & -0.3195 \\
\hline & $c_{2}$ & 4.4903 & 9.468 \\
\hline & $c_{3}$ & -1.1717 & -0.5022 \\
\hline & $c_{4}$ & 1.6498 & 1.590 \\
\hline & $c_{5}$ & -5.3579 & -4.893 \\
\hline & $c_{6}$ & 1.6327 & 1.200 \\
\hline
\end{tabular}

${ }^{a}$ The ErkV PEF has the same functional form as the ErkIV PEF and was not individually reparametrized. ${ }^{b}$ The deSPH/LJAT PEF has the same functional form as the PeaTHT PEF and was not individually reparametrized.

III.A-III.C, and the results are presented in Table 4. Also shown are the average MUE for the cluster data (labeled $\epsilon_{\mathrm{c}}$ ), i.e., the average of the errors over the five cluster data sets $\epsilon_{k}(k=1$, ..., 5); a "nonclose" cluster average labeled $\tilde{\epsilon}_{\mathrm{c}}$ that is explained in the next paragraph; and $\epsilon, E_{\mathrm{b}}$, and $a_{\mathrm{m}}$ that are defined in section II. The calculated $a_{\mathrm{m}}$ values in Table 4 may be compared to the experimental value ${ }^{19}$ of $4.022 \AA$. (Note that the experimental lattice constant corresponds to a bulk nearest-neighbor distance of $2.844 \AA$.) Most of the literature PEFs were parametrized for bulk properties, and many of the literature PEFs perform poorly for aluminum clusters. The average value of $\epsilon_{\mathrm{c}}$ for all of the literature PEFs is $1.7 \mathrm{eV} /$ atom, and no PEF from the literature has an MUE for the cluster data that is smaller than $0.12 \mathrm{eV} /$ atom.

We note that many of the literature PEFs fitted to experimental data were parametrized using values for the bulk cohesive energy and lattice constant determined at $298 \mathrm{~K}$ (3.39 $\mathrm{eV}$ and $4.050 \AA$, respectively). When the electronic energies computed using a PEF are compared to these experimental quantities, it is necessary to remove finite-temperature and zeropoint energy contributions, as discussed in ref 11 for the cohesive energy and in ref 19 for the lattice constant.

The four PEFs that are the least accurate in reproducing the aluminum cluster energies $\left(\epsilon_{\mathrm{c}} \geq 3.9 \mathrm{eV} /\right.$ atom $)$ are the HalP, ErkVIII, PeaTHT, and deSPH/LJAT PEFs, and they all employ a Lennard-Jones two-body interaction. This significant error is largely due to several geometries in the data set with compressed atom-atom distances for which the Lennard-Jones two-body interaction predicts very high energies, resulting in large errors. Certainly, an accurate representation of these geometries is important, but the error due to these geometries obscures the evaluation of the PEF for lower energy configurations. Therefore, the MUE over the cluster data was recomputed excluding geometries for which the smallest distance between any two atomic centers is less than $1.98 \AA$. (The distance $1.98 \AA$ corresponds to the bond distance in the accurate PBE0/MG3 $\mathrm{Al}_{2}$ curve at which the energy of the repulsive wall is equal to the energy of the dissociated atoms, i.e., it is the smallest classically allowed bond distance for bound $\mathrm{Al}_{2}$.) This error is denoted $\tilde{\epsilon}_{\mathrm{c}}$ and is also given in Table 4 . The errors are reduced significantly for the PEFs with Lennard-Jones two-body interactions, as well as for some of the other PEFs, when these compressed geometries are not considered, but the overall trends remain the same.

As expected, the PA PEFs are on average the least accurate group of PEFs, with an average value of $\epsilon_{\mathrm{c}}$ of $4.0 \mathrm{eV} /$ atom. The HalP PEF reproduces the bulk properties (against which it was parametrized) reasonably well but performs poorly over the cluster data set. The PetW PEF was derived entirely from theoretical considerations and was not fitted to any experimental or computed data. The PetW PEF predicts a reasonably accurate lattice constant, but the cohesive energy and cluster energies are qualitatively incorrect. The deSPH/M PEF was parametrized for $\mathrm{Al}_{6}$ clusters (which are not represented in our test set) and is the most accurate of the PA literature PEFs tested with $\epsilon_{\mathrm{c}}=$ $0.43 \mathrm{eV} /$ atom. The deSPH/M PEF is also reasonably accurate for the bulk data.

The NPA group of PEFs is more accurate than the PA group, with an average value of $\epsilon_{\mathrm{c}}$ of $2.8 \mathrm{eV} /$ atom. The best NPA PEF from the literature is the ErkIV PEF, which was parametrized to $\mathrm{Al}_{2}$ data as well as bulk data. Note that due to an error in the bulk data that was used during the original parametrization, ${ }^{61}$ the ErkIV PEF does not reproduce the bulk data used here. The ErkV PEF, although improved over the ErkIV PEF to include more bulk properties, does substantially worse for aluminum clusters.

The functional form for the ErkVIII, PeaTHT, and deSPH/ LJAT PEFs consists of a Lennard-Jones two-body interaction and an Axilrod-Teller three-body PEF. The ErkVIII PEF includes an additional three-body dispersion term. All three PEFs perform poorly for aluminum clusters, due in part to the presence of compressed atom-atom distances in the data set, as discussed above. However, even when these data are not considered, as in the $\tilde{\epsilon}_{\mathrm{c}}$ column, these PEFs do poorly for aluminum clusters. The PeaTHT and ErkVIII PEFs were parametrized using bulk data and reproduce the experimental lattice constant reasonably well but have large MUEs (greater than $4.1 \mathrm{eV} /$ atom) over the cluster data in the test set. The deSPH/LJAT PEF was parametrized using computed energies for several aluminum cluster sizes but does not perform well for either the clusters or the bulk data.

The CoxJM PEF has a general three-body functional form, and it performs well for the bulk data (to which it was parametrized) but does considerably less well for the cluster data, although the error is approximately reduced by half when geometries with compressed atom-atom distances are not considered.

The EA PEFs perform much better as a group than the PA and NPA groups of PEFs, with an average value of $\epsilon_{\mathrm{c}}$ of 0.42 
TABLE 4: MUEs (eV/atom) and FCC Lattice Constant $a_{\mathrm{m}}(\AA)$ for the Literature PEFs

\begin{tabular}{clcccccccccc}
\hline PEF type & \multicolumn{1}{c}{ PEF } & $\epsilon_{2}$ & $\epsilon_{3}$ & $\epsilon_{4}$ & $\epsilon_{7}$ & $\epsilon_{13}$ & $\epsilon_{\mathrm{c}}$ & $\tilde{\epsilon}_{\mathrm{c}}$ & $\epsilon_{\mathrm{b}}$ & $a_{\mathrm{m}}$ \\
\hline \multirow{2}{*}{ PA } & HalP & 4.396 & 16.64 & 6.641 & 22.89 & 0.596 & 10.23 & 1.783 & 0.162 & 8.449 & 4.05 \\
& PetW & 0.707 & 1.111 & 1.353 & 2.045 & 1.422 & 1.328 & 1.085 & 4.320 & 1.576 & 4.12 \\
& deSPH/M & 0.263 & 0.456 & 0.406 & 0.630 & 0.415 & 0.434 & 0.358 & 0.098 & 0.447 & 4.00 \\
NPA & ErkIV & 0.090 & 0.150 & 0.058 & 0.170 & 0.119 & 0.118 & 0.095 & 1.332 & 0.165 & 4.31 \\
& ErkV & 0.281 & 0.476 & 0.506 & 0.720 & 0.751 & 0.547 & 0.519 & 1.361 & 0.673 & 3.77 \\
& ErkVIII & 1.453 & 5.917 & 2.735 & 8.624 & 0.672 & 3.880 & 0.892 & 1.184 & 3.396 & 4.40 \\
& PeaTHT & 1.578 & 7.063 & 2.319 & 9.638 & 0.290 & 4.177 & 0.554 & 0.077 & 3.498 & 4.02 \\
& deSPH/LJAT & 2.830 & 12.00 & 4.348 & 16.80 & 0.382 & 7.270 & 1.034 & 0.807 & 6.079 & 4.27 \\
& CoxJM & 0.712 & 1.187 & 1.059 & 1.854 & 0.227 & 1.008 & 0.478 & 0.007 & 0.839 & 4.00 \\
EA & Gol & 0.127 & 0.122 & 0.073 & 0.149 & 0.132 & 0.121 & 0.098 & 0.099 & 0.126 & 4.05 \\
& BetH & 0.311 & 0.300 & 0.284 & 0.196 & 0.093 & 0.237 & 0.210 & 0.059 & 0.177 & 4.03 \\
& CleR & 0.517 & 0.636 & 0.702 & 0.783 & 0.251 & 0.578 & 0.438 & 0.940 & 0.514 & 3.55 \\
& PapCEP & 0.137 & 0.203 & 0.133 & 0.340 & 0.156 & 0.194 & 0.136 & 0.017 & 0.195 & 3.96 \\
& PapKEP & 0.442 & 0.960 & 0.605 & 1.585 & 0.250 & 0.768 & 0.293 & 0.142 & 0.689 & 3.93 \\
& SutC & 0.565 & 0.613 & 0.611 & 0.470 & 0.183 & 0.488 & 0.426 & 0.075 & 0.372 & 4.04 \\
& MeiD & 0.552 & 0.567 & 0.534 & 0.380 & 0.242 & 0.455 & 0.439 & 0.035 & 0.359 & 4.05 \\
& StrM & 0.388 & 0.438 & 0.389 & 0.373 & 0.098 & 0.337 & 0.249 & 0.023 & 0.252 & 4.04 \\
& MisFMP & 0.313 & 0.613 & 0.451 & 0.872 & 0.091 & 0.468 & 0.194 & 0.035 & 0.386 & 4.00 \\
& Jac & 0.885 & 0.701 & 0.474 & 0.334 & 0.427 & 0.564 & 0.560 & 0.161 & 0.461 & 3.94
\end{tabular}

TABLE 5: MUEs (eV/atom) and FCC Lattice Constant $a_{\mathrm{m}}(\AA)$ for the Reparametrized PEFs

\begin{tabular}{|c|c|c|c|c|c|c|c|c|c|c|c|}
\hline PEF type & PEF & $\epsilon_{2}$ & $\epsilon_{3}$ & $\epsilon_{4}$ & $\epsilon_{7}$ & $\epsilon_{13}$ & $\epsilon_{\mathrm{c}}$ & $\tilde{\epsilon}_{\mathrm{c}}$ & $\epsilon_{\mathrm{b}}$ & $\epsilon$ & $a_{\mathrm{m}}$ \\
\hline \multirow[t]{3}{*}{$\mathrm{PA}$} & HalP & 0.339 & 0.588 & 0.705 & 0.983 & 1.158 & 0.755 & 0.757 & 3.132 & 1.011 & 3.91 \\
\hline & PetW & 0.302 & 0.354 & 0.352 & 0.243 & 0.208 & 0.292 & 0.266 & 1.700 & 0.306 & 3.92 \\
\hline & deSPH/M & 0.194 & 0.394 & 0.356 & 0.575 & 0.118 & 0.327 & 0.230 & 2.028 & 0.353 & 3.99 \\
\hline \multirow[t]{4}{*}{ NPA } & ErkIV $^{a}$ & 0.125 & 0.242 & 0.090 & 0.101 & 0.058 & 0.123 & 0.097 & 1.020 & 0.127 & 4.20 \\
\hline & ErkVIII & 0.281 & 0.344 & 0.242 & 0.187 & 0.045 & 0.220 & 0.158 & 2.680 & 0.238 & 3.94 \\
\hline & $\mathrm{LJAT}^{b}$ & 0.290 & 0.388 & 0.255 & 0.264 & 0.049 & 0.249 & 0.164 & 3.193 & 0.282 & 3.95 \\
\hline & CoxJM & 0.123 & 0.132 & 0.118 & 0.071 & 0.031 & 0.095 & 0.089 & 0.157 & 0.072 & 4.48 \\
\hline \multirow[t]{7}{*}{ EA } & Gol & 0.086 & 0.068 & 0.076 & 0.062 & 0.027 & 0.063 & 0.058 & 0.674 & 0.071 & 3.86 \\
\hline & $\mathrm{GEA}^{c}$ & 0.187 & 0.152 & 0.140 & 0.085 & 0.075 & 0.128 & 0.119 & 0.008 & 0.099 & 3.93 \\
\hline & SutC & 0.277 & 0.268 & 0.200 & 0.150 & 0.122 & 0.204 & 0.171 & 0.161 & 0.165 & 4.07 \\
\hline & MeiD & 0.047 & 0.063 & 0.088 & 0.047 & 0.028 & 0.056 & 0.053 & 0.001 & 0.046 & 4.00 \\
\hline & StrM & 0.058 & 0.064 & 0.084 & 0.050 & 0.038 & 0.059 & 0.058 & 0.079 & 0.052 & 3.84 \\
\hline & MisFMP & 0.089 & 0.079 & 0.094 & 0.044 & 0.032 & 0.068 & 0.069 & 0.000 & 0.051 & 4.05 \\
\hline & $\mathrm{Jac}$ & 0.195 & 0.144 & 0.092 & 0.077 & 0.040 & 0.110 & 0.097 & 0.871 & 0.104 & 3.97 \\
\hline
\end{tabular}

${ }^{a}$ The ErkV PEF has the same functional form as the ErkIV PEF. ${ }^{b}$ Lennard-Jones/Axilrod-Teller functional form. The PeaTHT and deSPH/ LJAT PEFs both use this functional form. ${ }^{c}$ The general EA functional form with exponential embedding function given in eqs 31 and 32 with $n$ $=1 / 2$. The BetH, CleR, PapCEP, and PapKEP PEFs use this functional form or an equivalent one.

eV/atom. All of the EA PEFs were fitted to bulk properties, and all but one (CleR) predicts reasonably accurate lattice constants and cohesive energies. (The poor performance of the CleR PEF could indicate a typographical error in the published parameters for the CleR PEF; the original authors indicate that the CleR PEF predicts these quantities accurately for aluminum. ${ }^{57}$ ) The cluster energies are reproduced with varying accuracy for the various EA PEFs, and the errors are not very sensitive to whether geometries with compressed atom-atom distances are included in the test set. The best EA PEF from the literature is the Gol PEF with $\epsilon_{\mathrm{c}}=0.12 \mathrm{eV} / \mathrm{atom}$.

None of the analytic PEFs obtained from the literature performs accurately enough for aluminum clusters to allow for quantitative work. Furthermore, it is clearly dangerous to employ PEFs for studies outside of their intended range of application.

IV.B. Reparametrized Literature PEFs. The nineteen literature PEFs discussed above were used for a variety of different purposes and parametrized against a variety of different data. It is not possible, therefore, to infer the success or failure of any particular functional form based on the errors in Table 4. To study the relative flexibility of the various functional forms themselves, the literature PEFs were reparametrized by minimizing the total MUE ( $\epsilon$ in eq 8) with respect to the available adjustable parameters. The reoptimized parameters are listed in Tables 1-3, and the MUEs for the reparametrized PEFs are given in Table 5. Note that some of the eighteen PEFs from the literature have equivalent functional forms, as indicated in Tables 2, 3, and 5 .

Optimizing the parameters for each PEF results in MUEs over the cluster data in the test set that are up to $97 \%$ smaller than those for the original PEFs from the literature, with an average improvement of $70 \%$. All three classes of PEFs (PA, NPA, and EA) improve by roughly the same amount overall, with slightly better improvement for the NPA group. Due to the intentionally low weight given to the bulk properties, the errors in the bulk properties occasionally increase upon reparametrization.

As seen from Table 5, the three PA PEFs are the least accurate reparametrized PEFs overall (with $\epsilon_{\mathrm{c}} \geq 0.33 \mathrm{eV} /$ atom), clearly demonstrating that an effective two-body term is unable to model clusters of varying sizes with quantitative accuracy.

The NPA group is the second most accurate group of reparametrized PEFs. Note that the PeaTHT and deSPH/LJAT PEFs have identical functional forms, and this functional form is labeled LJAT in Table 5. The most accurate reparametrized NPA PEF (and fifth best reparametrized PEF overall) is the CoxJM PEF with $\epsilon_{\mathrm{c}}=0.095 \mathrm{eV} /$ atom. The widely used LJAT PEF performs poorly for aluminum clusters, and the additional term in the ErkVIII PEF provides only a modest improvement in $\epsilon$ (and no improvement in $\epsilon_{\mathrm{c}}$ ) over the LJAT approach.

Next we consider the three simplest EA PEFs: the Gol, GEA (which labels the common functional form used in the BetH, CleR, PapCEP, and PapKEP PEFs), and SutC PEFs. The SutC 
PEF uses an embedding function involving inverse powers of the bond distance, and Table 5 shows that this approach is less accurate than the Gol and GEA approaches that use exponentialbased embedding functions. The Gol and GEA PEFs differ only in that the Gol PEF allows the exponent in the embedding function ( $n$ in eq 32) to vary, whereas for the GEA PEF, $n=$ $1 / 2$. This additional flexibility results in a $51 \%$ smaller cluster MUE for the Gol PEF as compared to the GEA PEF. It is interesting to note that the GEA and Gol PEFs, although very similar in their functional forms, have qualitatively different distributions of errors over the data set. The Gol PEF does well for the cluster energies and poorly for the bulk data, whereas the situation is reversed for the GEA PEF, demonstrating the ambiguity in finding the "best" set of parameters that minimizes a particular error function. Clearly, more than one set of parameters that gives a "small" error may be found with varying error distributions.

The three best reparametrized PEFs overall are the MeiD, StrM, and MisFMP PEFs, which have impressively small total MUEs of only $\sim 0.05 \mathrm{eV} /$ atom. For all three PEFs, the error is fairly evenly distributed over the data in the test set, but the MeiD and StrM PEFs are slightly more accurate for the cluster data. The StrM PEF uses the same embedding function as the GEA PEF but has a more highly parametrized two-body interaction. This additional flexibility allows for a total MUE that is $\sim 50 \%$ smaller than that of the GEA PEF. The best reparametrized PEF overall is the MeiD PEF, which performs well for both the cluster and bulk data.

IV.C. Many-Body Effects. The most accurate reparametrized PEFs identified in section IV.B (the Gol, MeiD, MisFMP, and StrM PEFs) could be used with some confidence to model aluminum clusters. It is not clear, however, how well these PEFs would perform (i.e., how transferable the reoptimized parameters would be) for systems similar to but not explicitly represented in the data set. Therefore, in this section a systematic examination of the ability of several of the functional forms discussed above to model many-body effects in general is presented.

Many-body effects are especially important for clusters and nanoparticles. In the bulk metal and in the absence of defects, vacancies, and dislocations, every atom has the same number of neighbors, which, moreover, are distributed uniformly in space. In clusters, however, atoms experience a greater variety of exposure to the surface, and hence a larger fraction of these atoms have coordination numbers lower than in the bulk. Table 6 shows the coordination number distribution of the atoms in several quasispherical clusters. For clusters with diameters $d \leq$ $5 \mathrm{~nm}$, greater than $30 \%$ of atoms are surface atoms, and there is a significant distribution of coordination numbers. If second-, third-, etc. nearest neighbors are considered, there is even greater diversity in atomic environments. Furthermore, when modeling the dynamics of energetic processes such as collisions, combustion, or cluster dynamics, high-energy configurations, which involve large displacements from the bulk lattice sites, may be important.

As a first step toward understanding many-body effects in aluminum clusters, the $\mathrm{Al}_{2}$ data in our test set was used to parametrize the extended Rydberg two-body functional form discussed in section III.A, with linear scaling introduced using the cutoff function in eq 70. Cutoff functions are often introduced after the functional form has been fitted (such as when the PEF is used during a simulation), and the parameters of the cutoff function are chosen such that the magnitude of the cutoff function is close to unity (or exactly unity if a step function is used) when the magnitude of the PEF is appreciable
TABLE 6: Percentage of Atoms Having $m=3, \ldots, 12$ Nearest Neighbors in Several FCC Quasispherical Clusters (QSCs) of Size $N$ and Approximate Diameter $d$

\begin{tabular}{rccrrrrrrrrr}
\hline & & \multicolumn{10}{c}{$m$} \\
\cline { 3 - 12 }$N$ & $d(\mathrm{~nm})^{a}$ & 3 & 4 & 5 & 6 & 7 & 8 & 9 & 10 & 11 & 12 \\
\hline 13 & 0.9 & 0 & 0 & 92 & 0 & 0 & 0 & 0 & 0 & 0 & 8 \\
19 & 1.1 & 0 & 32 & 0 & 0 & 63 & 0 & 0 & 0 & 0 & 5 \\
43 & 1.3 & 0 & 0 & 56 & 0 & 0 & 14 & 0 & 0 & 28 & 2 \\
55 & 1.4 & 0 & 0 & 22 & 0 & 44 & 11 & 0 & 0 & 0 & 24 \\
79 & 1.6 & 0 & 0 & 0 & 30 & 15 & 0 & 30 & 0 & 0 & 24 \\
87 & 1.7 & 9 & 0 & 0 & 28 & 14 & 0 & 0 & 28 & 0 & 22 \\
135 & 1.8 & 0 & 0 & 0 & 36 & 0 & 18 & 6 & 0 & 9 & 32 \\
141 & 1.9 & 0 & 4 & 0 & 34 & 0 & 0 & 23 & 0 & 9 & 30 \\
177 & 2.0 & 0 & 0 & 20 & 0 & 0 & 31 & 5 & 0 & 14 & 31 \\
675 & 3.0 & 0 & 0 & 0 & 7 & 11 & 8 & 14 & 0 & 5 & 55 \\
1601 & 4.0 & 0 & 0 & 0 & 6 & 8 & 4 & 10 & 1 & 6 & 63 \\
3367 & 5.0 & 0 & 0 & 1 & 6 & 5 & 4 & 7 & 3 & 6 & 70 \\
5979 & 6.0 & 0 & 0 & 0 & 5 & 4 & 4 & 4 & 4 & 4 & 75 \\
9693 & 7.0 & 0 & 0 & 0 & 4 & 4 & 2 & 5 & 3 & 4 & 78 \\
14363 & 8.0 & 0 & 0 & 0 & 3 & 4 & 3 & 4 & 3 & 4 & 81
\end{tabular}

${ }^{a}$ The diameter $d$ is calculated as $R_{\max }+R_{\mathrm{nn}}$ where $R_{\max }$ is the largest interatomic distance in the cluster and $R_{\mathrm{nn}}$ is the nearest neighbor distance for bulk aluminum.

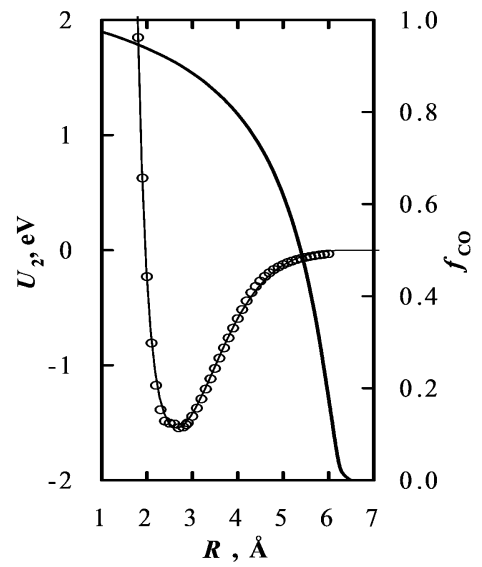

Figure 1. Accurate and fitted two-body interaction for $\mathrm{Al}_{2}$. The PBE0/ MG3 data are shown as circles; the fitted data for the ER2 PEF are shown as a thin solid line. The cutoff function $f_{\mathrm{CO}}$ used in the ER2 $\mathrm{PEF}$ is shown as a thick solid line.

and goes to zero only when the magnitude of the PEF is negligible. This approach requires that convergence with respect to the cutoff distance must be demonstrated.

To avoid this future complication, the cutoff function is built into the functional form, and the parameters of the two-body term $U_{2}$ are optimized along with those in $f_{\mathrm{CO}}$. This approach, in general, results in smaller cutoff thresholds, while retaining smooth and accurate functional forms. Specifically, the five parameters in $U_{2}$ (see eqs 18 and 19) were optimized simultaneously with the range parameter $\Delta$ in the cutoff function in eq 70 . The cutoff distance $R_{\mathrm{CO}}$ was not allowed to vary during fitting, and several values of $R_{\mathrm{CO}}$ were tested from 5.5 to 7.0 $\AA$. We found that $R_{\mathrm{CO}}=6.5 \AA$ is the minimum distance at which the error in the $\mathrm{Al}_{2}$ data $\epsilon_{2}$ was less than $0.015 \mathrm{eV} /$ atom and the potential remained smooth. This PEF (fitted to $\mathrm{Al}_{2}$ data only) is labeled ER2. The fitted ER2 energy curve for $\mathrm{Al}_{2}$, the accurate PBE0/MG3 energies for $\mathrm{Al}_{2}$, and the cutoff function used in the ER2 PEF is shown in Figure 1, the MUEs for the ER2 PEF are summarized in Table 7, and the parameters for the ER2 PEF are in Table 8.

Note that the isolated $\mathrm{Al}_{2}$ curve involves a crossing of the ${ }^{3} \Pi_{\mathrm{u}}$ and ${ }^{3} \Sigma_{\mathrm{g}}^{-}$electronic states near the equilibrium distance for both curves, as discussed elsewhere ${ }^{68}$ and as seen in the accurate 
TABLE 7: MUEs (eV/atom) and FCC Lattice Constant $a_{\mathrm{m}}(\AA)$ for the Many-Body PEFs

\begin{tabular}{|c|c|c|c|c|c|c|c|c|c|c|}
\hline PEF & $\epsilon_{2}$ & $\epsilon_{3}$ & $\epsilon_{4}$ & $\epsilon_{7}$ & $\epsilon_{13}$ & $\epsilon_{\mathrm{c}}$ & $\tilde{\epsilon}_{\mathrm{c}}$ & $\epsilon_{\mathrm{b}}$ & $\epsilon$ & $a_{\mathrm{m}}$ \\
\hline ER2 & 0.013 & 0.138 & 0.420 & 0.951 & 1.322 & 0.569 & 0.552 & 17.022 & 1.433 & 2.66 \\
\hline ER & 0.239 & 0.405 & 0.427 & 0.319 & 0.133 & 0.305 & 0.292 & 2.081 & 0.315 & 3.95 \\
\hline $\mathrm{ER} 2+\mathrm{AT}$ & 0.013 & 0.149 & 0.425 & 0.617 & 0.648 & 0.370 & 0.310 & 4.580 & 0.650 & 4.14 \\
\hline $\mathrm{ER} 2+\mathrm{EAT}$ & 0.013 & 0.149 & 0.426 & 0.612 & 0.651 & 0.370 & 0.311 & 4.596 & 0.650 & 4.13 \\
\hline ER2+CoxJM & 0.013 & 0.091 & 0.181 & 0.246 & 0.258 & 0.158 & 0.148 & 0.866 & 0.232 & 4.44 \\
\hline ER2+GEA & 0.013 & 0.113 & 0.115 & 0.092 & 0.042 & 0.075 & 0.060 & 0.005 & 0.067 & 3.98 \\
\hline ER2+MeiD & 0.013 & 0.079 & 0.100 & 0.199 & 0.027 & 0.083 & 0.070 & 0.276 & 0.089 & 4.14 \\
\hline ER2+MisFMP & 0.013 & 0.124 & 0.188 & 0.371 & 0.129 & 0.165 & 0.070 & 0.002 & 0.181 & 4.05 \\
\hline $\mathrm{ER} 2+\mathrm{S}$ & 0.013 & 0.075 & 0.099 & 0.140 & 0.067 & 0.079 & 0.059 & 0.028 & 0.084 & 3.83 \\
\hline $\mathrm{ER} 2+\mathrm{ES}$ & 0.013 & 0.081 & 0.108 & 0.095 & 0.057 & 0.071 & 0.056 & 0.007 & 0.071 & 4.05 \\
\hline $\mathrm{ER} 2+\mathrm{BA}$ & 0.013 & 0.136 & 0.406 & 0.859 & 1.131 & 0.509 & 0.492 & 4.683 & 0.915 & 4.15 \\
\hline ER2+EBA & 0.013 & 0.108 & 0.188 & 0.092 & 0.077 & 0.095 & 0.089 & 0.020 & 0.092 & 4.15 \\
\hline $\mathrm{ER} 2+\mathrm{CN}$ & 0.013 & 0.082 & 0.125 & 0.250 & 0.032 & 0.100 & 0.060 & 0.003 & 0.098 & 3.97 \\
\hline $\mathrm{ER} 2+\mathrm{ECN}$ & 0.013 & 0.085 & 0.095 & 0.077 & 0.030 & 0.060 & 0.054 & 0.006 & 0.053 & 4.04 \\
\hline ER2+EACN & 0.013 & 0.094 & 0.096 & 0.091 & 0.028 & 0.064 & 0.055 & 0.007 & 0.057 & 4.04 \\
\hline $\mathrm{ER} 2+\mathrm{SCNm}$ & 0.013 & 0.073 & 0.102 & 0.107 & 0.032 & 0.066 & 0.054 & 0.042 & 0.062 & 4.15 \\
\hline ER2+ESCNm & 0.013 & 0.075 & 0.096 & 0.072 & 0.028 & 0.057 & 0.054 & 0.001 & 0.050 & 4.06 \\
\hline $\mathrm{ER} 2+\mathrm{SCNa}$ & 0.013 & 0.076 & 0.102 & 0.141 & 0.026 & 0.072 & 0.053 & 0.177 & 0.072 & 4.15 \\
\hline $\mathrm{ER} 2+\mathrm{ESCNa}$ & 0.013 & 0.080 & 0.087 & 0.063 & 0.027 & 0.054 & 0.048 & 0.001 & 0.047 & 4.04 \\
\hline
\end{tabular}

electronic structure data in Figure 1. We chose not to fit these data to two curves, but rather to a curve that is smooth for all values of the bond distance. The fitted equilibrium bond distance $2.65 \AA$ is slightly smaller than the experimental value ${ }^{70,71}$ of $2.70 \AA$ due to this effect. The dissociation energy was not allowed to exceed $1.55 \mathrm{eV}$ during the fitting procedure.

The extended Rydberg functional form provided a good fit to the two-body interaction $\left(\epsilon_{2}=0.013 \mathrm{eV} /\right.$ atom $)$, and other functional forms for the diatomic curve were not pursued. It can be seen from Table 7 that the ER2 PEF performs poorly for all but the $\mathrm{Al}_{2}$ clusters, clearly demonstrating the presence of many-body effects in our data set.

For comparison, the extended Rydberg functional form with a $6.5 \AA$ cutoff was parametrized against the entire data set, and this PEF is labeled ER in Tables 7 and 8, where the errors and parameters are given. The total MUE for the ER PEF is 0.32 eV/atom, which is slightly more accurate than the deSPH/M PEF, the most accurate PA PEF discussed in section IV.B. The extended Rydberg functional form, although quite flexible, is not able to accurately fit the complicated many-body effects present in the data set with an effective two-body interaction.

An analytic fit to the accurate two-body interaction allows for a systematic study of the effectiveness of the various functional forms discussed in section III in modeling manybody effects. First, the ER2 two-body PEF was paired with three of the three-body terms from section III.D: the AT term in eq 64, the extended AT (EAT) approach in eq 61, and the general three-body term of Murrell (CoxJM) shown in eq 67. Parameters for the three-body terms were obtained by minimizing the error in eq 8 while keeping the parameters for the two-body interaction fixed at their ER2 values. Cutoff functions were introduced into the three-body terms, as shown in eq 72, with a cutoff distance of $6.5 \AA$. The range parameter $\Delta$ in eq 70 was allowed to vary. (Note: The highly flexible three-body term in the CoxJM functional form gives unphysical oscillations in the cohesive energy as a function of lattice constant when parametrized using the present data set and the accurate two-body interaction. We therefore truncated eq 67 at terms that are second-order in the bond distances; i.e., $c_{4}, c_{5}$, and $c_{6}$ were set to zero. The resulting optimized total MUE for the CoxJM PEF did not change significantly, and oscillations in the bulk cohesive energy curve were reduced. We also leave out the damping function in eq 69 due to the inclusion of the cutoff functions.) The MUEs for these many-body PEFs are shown in Table 7 where the PEFs are labeled ER2+AT, ER2+EAT, and
ER2+CoxJM. Parameters for these many-body PEFs are tabulated in Table 8.

The AT term, paired with the accurate two-body curve, reduces the average error in the cluster data by $\sim 37 \%$ (as compared with the ER2 PEF) but does not provide quantitative accuracy. The addition of the next-leading term (as in the EAT PEF) has a negligible effect on the results. The three-body term of Murrell and co-workers (CoxJM) is highly flexible and reduces the error in the cluster data by $72 \%$ to $0.16 \mathrm{eV} /$ atom.

Next we consider the general embedded atom function (GEA) given in eq 32 as well as the more complicated embedding functions of Mei and Davenport (MeiD) shown in eqs 38 and 39 and Mishin et al. (MisFMP) shown in eqs 45-47. Note that the embedding function contains two-body interactions. We wish to isolate the embedding function's ability to model many-body effects, so the two-body part of the embedding function is subtracted from the full embedding function; e.g., the manybody part of eq 32 is

$$
F_{\alpha}^{\mathrm{MB}}=-B\left(\sum_{\beta \neq \alpha} \exp \left(-b R_{\alpha \beta}\right)\right)^{n}+B \sum_{\beta \neq \alpha}\left[\exp \left(-b R_{\alpha \beta}\right)\right]^{n}
$$

Cutoff functions were added as in eq 73 with a cutoff distance of $6.5 \AA$. The many-body part of the GEA, MeiD, and MisFMP PEFs were combined with the accurate two-body expression ER2, and the many-body and cutoff range parameter were optimized, holding the two-body parameters fixed. The MUEs for the optimized ER2+GEA, ER2+MeiD, and ER2+MisFMP PEFs are given in Table 7, and the optimized parameters are tabulated in Table 8 .

The optimized GEA many-body term has an average MUE for the cluster data of $0.075 \mathrm{eV} /$ atom with smaller errors for the larger clusters and the bulk data. The MisFMP and MeiD PEFs, although they have more adjustable parameters, do not perform as well as the simpler GEA form.

Finally, we consider the screening, bond angle, and coordination number many-body functions introduced in section III.B as implemented using eq 20 and the extended formalism in eq 21 and using the ER2 two-body interaction. Linear scaling is introduced using eq 70 with a cutoff distance of $6.5 \AA$. Parameters for all of the many-body PEFs are tabulated in Table 8. [When the extended formalism is used (eq 21), the parameters for $U_{2}^{\prime}$ are denoted with primes in Table 8.] The MUEs for these PEFs are given in Table 7.

Five of the six functional forms designed to model specific many-body effects (i.e., the S, ES, EBA, CN, and ECN 
TABLE 8: Parameters for the Many-Body PEFs

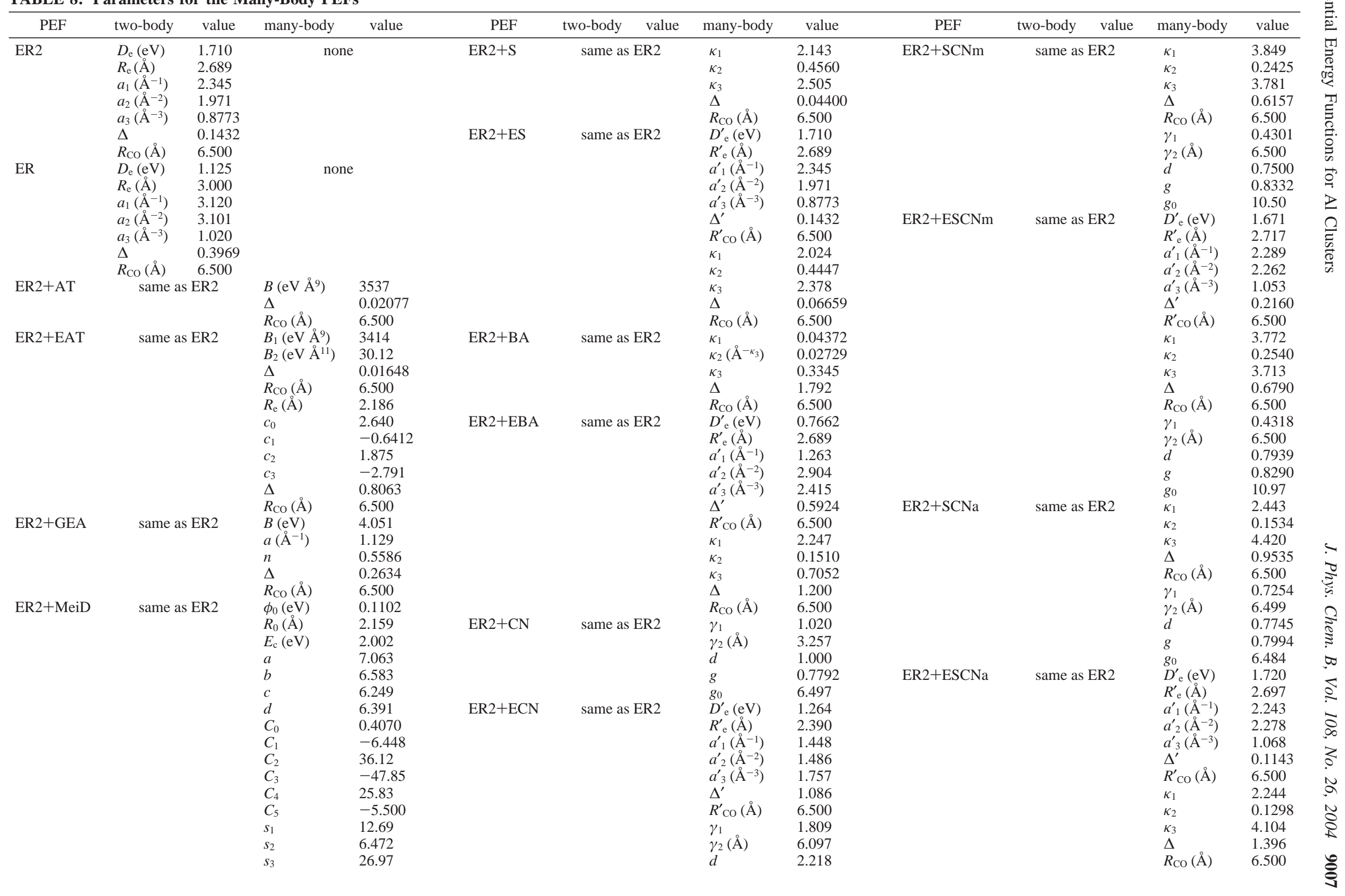




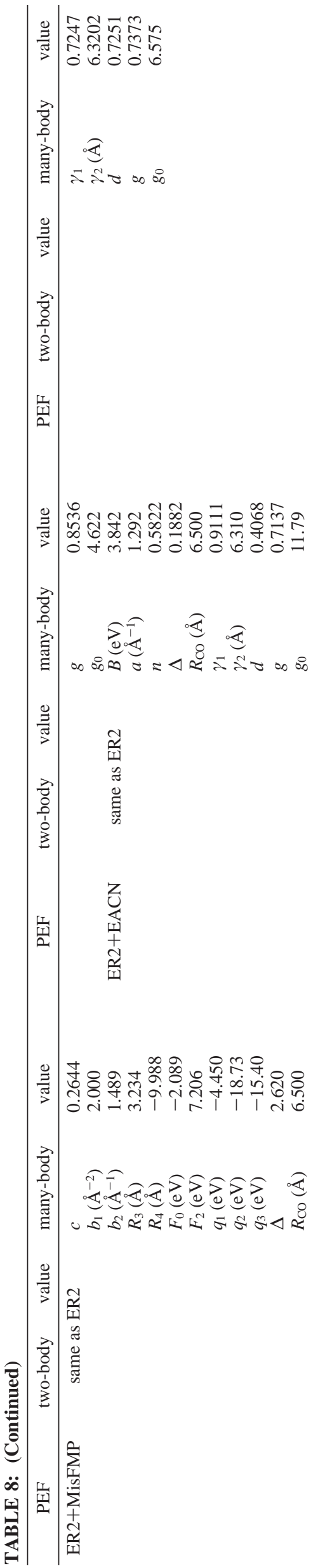

functional forms) perform well overall; the sixth, BA, term performs poorly, but the remaining five terms all have an average MUE for the cluster data that is less than $\sim 0.10 \mathrm{eV} /$ atom. The $\mathrm{CN}$ term gives the smallest error for the bigger clusters (which have the largest contribution to their total energies from coordination effects), whereas the error for the $\mathrm{S}$ term is more evenly distributed. The BA function, which was designed to be more flexible than the screening function, does not predict an accurate bulk cohesive energy and is much less accurate overall than the simpler $\mathrm{S}$ function. The extended version of the $\mathrm{S}$ functional form involves six additional parameters but does not significantly improve the error in the cluster data. The extended version of the BA functional form, however, greatly reduces the error. This result may be interpreted to indicate that the functional form of the $\mathrm{S}$ term is physically motivated, whereas the BA term is not. The extended version of the $\mathrm{CN}$ term shows a modest improvement over the $\mathrm{CN}$ term for the smaller clusters.

We developed and evaluated several PEFs in which the accurate ER2 two-body term was combined with two or more of the many-body effects discussed above. The most accurate PEF that consisted of the ER2 two-body term in combination with the EA and one other many body term is the ER2+EACN PEF, where

$$
V=\sum_{\alpha>\beta} U_{2}\left(R_{\alpha \beta}\right) f_{\alpha \beta}^{\mathrm{CN}}+\sum_{\alpha} F_{\alpha}^{\mathrm{MB}}
$$

These errors and parameters for the ER2+EACN PEF are listed in Tables 7 and 8, respectively.

It is pleasing that the physically motivated and fairly simple $\mathrm{S}$ and $\mathrm{CN}$ functional forms are able to modify the accurate twobody interaction such that the overall errors are on the order of the best optimized PEFs discussed in section IV.D and that the trends in the errors for the cluster data support their physical interpretation. Therefore, we combine the $\mathrm{S}$ and $\mathrm{CN}$ approaches and define two new many-body PEFs. Specifically, the two terms may be combined multiplicatively as in the SCNm PEF, where

$$
V=\sum_{\alpha>\beta} U_{2}\left(R_{\alpha \beta}\right)-\sum_{\alpha>\beta} U_{2}^{\prime}\left(R_{\alpha \beta}\right)\left(1-f_{\alpha \beta}^{\mathrm{S}}\right)\left(1-f_{\alpha \beta}^{\mathrm{CN}}\right)
$$

or additively as in the SCNa PEF, where

$$
\begin{gathered}
V=\sum_{\alpha>\beta} U_{2}\left(R_{\alpha \beta}\right)-\sum_{\alpha>\beta} U_{2}^{\prime}\left(R_{\alpha \beta}\right)\left(1-f_{\alpha \beta}^{\mathrm{S}}\right)- \\
\sum_{\alpha>\beta} U_{2}^{\prime}\left(R_{\alpha \beta}\right)\left(1-f_{\alpha \beta}^{\mathrm{CN}}\right)
\end{gathered}
$$

Note that both the SCNm and SCNa methods may be implemented with both $U_{2}$ and $U_{2}^{\prime}$ set equal to the accurate ER2 two-body interaction (these two PEFs are labeled ER2 + SCNm and ER2+SCNa), or with $U_{2}$ equal to the ER2 two-body interaction and with $U_{2}^{\prime}$ allowed to vary during fitting (these two PEFs are labeled ER2+ESCNm and ER2+ESCNa). The MUEs for these four new PEFs are included in Table 7, and the optimized parameters are in Table 8 .

In general, the four PEFs show reduced errors as compared with the PEFs that include only one or the other of the $S$ or the $\mathrm{CN}$ function. The ER2+ESCNa PEF is the best overall of the PEFs featuring an accurate two-body interaction with a total MUE of $0.047 \mathrm{eV} /$ atom, a MUE for the cluster data of 0.054 eV/atom, and a MUE for the bulk data of $0.001 \mathrm{eV} / \mathrm{atom}$. To verify the validity of the extrapolation in eq 1 , the energies of 
27 QSCs from $\mathrm{Al}_{13}$ to $\mathrm{Al}_{3043}$ were computed using the ER2+ESCNa PEF. The bulk cohesive energy estimated using eq $1(3.43 \mathrm{eV})$ agrees well with the bulk cohesive energies computed from a least-squares fit to all 27 data points (3.44 $\mathrm{eV}$ ) and from a least-squares fit including only those clusters whose cohesive energies differ from the limiting value by less than $50 \%$ of the difference between the limiting value and the cohesive energy of $\mathrm{Al}_{13}(3.42 \mathrm{eV})$.

Finally, we tested the effect of allowing the two-body term to vary in addition to the three-body terms for all of the PEFs in Table 7. This strategy resulted in slightly smaller overall errors for some of the PEFs. The "best" PEFs had errors comparable to or slightly larger than those for the ER2+ESCNa $\mathrm{PEF}$, and so we do not consider this approach any further.

Overall, the best analytic PEF for aluminum clusters is the ER2+ESCNa PEF, which features linear scaling, physically motivated screening and coordination number terms, an average error of $0.05 \mathrm{eV} /$ atom in the cluster data, and accurate twobody and bulk limits.

It is interesting to compare the performance of the methods examined here to the performance of semiempirical molecular orbital methods of the neglect-of-differential-overlap and tightbinding types, as studied in ref 11 . That reference, which used a representative subset of the present test set, found mean unsigned errors per atom of $0.25-3.64 \mathrm{eV}$ for the six methods tested, and only AM1 ${ }^{72,73}$ had a mean unsigned error per atom of less than $0.41 \mathrm{eV}$. The methods examined here have mean unsigned errors per atom that are 5 and 8 times more accurate than the best (AM1) and second best (MSINDO ${ }^{74,75}$ ) of these standard molecular orbital methods, respectively. Furthermore, the molecular orbital methods are $\sim 9000$ (AM1) and $\sim 20000$ (MSINDO) times more expensive than the analytic PEFs for a single $\mathrm{Al}_{13}$ energy calculation, and relatively even more expensive for larger systems.

\section{Conclusions}

Several potential energy functions (PEFs) from the literature that have been previously parametrized for aluminum have been collected and tested using a data set composed of 224 aluminum cluster energies and the FCC cohesive energy and lattice constant. Several different functional forms were tested, including pairwise additive (PA), nonpairwise additive (NPA) with two- and three-body terms, and embedded atom (EA) PEFs. The two best literature PEFs have total mean unsigned errors (MUEs) averaged over the clusters in the test set of $0.12 \mathrm{eV} /$ atom.

All of the PEFs from the literature have been reparametrized against the data in the test set, resulting in five PEFs with MUEs for the cluster data of less than $0.10 \mathrm{eV} /$ atom. Pairwise additive PEFs are incapable of modeling the complicated many-body effects found in clusters of various sizes. The best literature PEF with an explicit three-body term includes the general polynomial form of Murrell and co-workers and has a MUE for the cluster data of $0.10 \mathrm{eV} /$ atom. The widely used LennardJones two-body term coupled with the Axilrod-Teller dispersion term is incapable of accurately modeling aluminum clusters and has a total MUE of $0.25 \mathrm{eV} /$ atom. The reparametrized embedded-atom PEFs perform well, and the best reparametrized EA PEFs have MUEs of $0.06 \mathrm{eV} /$ atom.

Finally, a systematic study of the ability of various functional forms to model many-body effects has been presented. An accurate fit to the diatomic molecule is presented and is used to test the ability of several functional forms to correct the twobody interaction in the presence various atomic environments.
The AT term is shown to be inaccurate for aluminum clusters in this context as well. The screening function of Ho et al. and the newly presented coordination number function both perform well, and the results indicate that these functions incorporate the important physical features. The best analytic potential overall has a MUE over the cluster data of $0.05 \mathrm{eV} /$ atom and includes screening and coordination number effects, linear scaling, and accurate two-body and bulk limits. This PEF, labeled ER2+ESCNa, would be very efficient for dynamics simulations of Al clusters, nanoparticles, and solids.

Acknowledgment. This work was supported in part by the Defense-University Research Initiative in Nanotechnology (DURINT) through a grant managed by the Army Research Office.

\section{References and Notes}

(1) Born, M..; Oppenheimer, R. Ann. Phys. 1927, 84, 457.

(2) Born, M.; Huang, K. The Dynamical Theory of Crystal Lattices; Oxford University Press: London, 1954.

(3) Kohn, W.; Becke, A. D.; Parr, R. G., J. Phys. Chem. 1996, 100, 12974

(4) Perdew, J. P. In Density Functional Theory: A Bridge Between Chemistry and Physics; Geelings, P., DeProft, F., Laugenaeker, W., Eds.; Free University of Brussels Press: Brussels, 1998; p 87.

(5) Melius, C. F.; Goddard, W. A., III. Phys. Rev. 1974, A10, 1528. 3826.

(6) Kahn, L. R.; Baybutt, P.; Truhlar, D. G. J. Chem. Phys. 1976, 65,

(7) Krauss, M.; Stevens, W. J. Annu. Rev. Phys. Chem. 1984, 35, 357.

(8) Ahlrics, R.; Elliot, S. D. Phys. Chem. Chem. Phys. 1999, 1, 13.

(9) Schultz, N. E.; Truhlar, D. G. Manuscript in preparation.

(10) Tight-Binding Approach to Computational Materials Science; Turchi, P. E. A., Gonis, A., Colombo, L., Eds.; Materials Research Society: Warrendale, PA, 1998.

(11) Schultz, N. E.; Staszewska, G.; Staszewski, P.; Truhlar, D. G. J. Phys. Chem. B 2004, 108, 4143.

(12) Perdew, J. P.; Burka, K.; Ernzerhof, M. Phys. Rev. Lett. 1996, 77, 3865; 1997, 78, 1396(E).

(13) Ernzerhof, M.; Scuseria, G. E. J. Chem. Phys. 1999, 110, 5029.

(14) Adamo, C.; Barone, V. J. Chem. Phys. 1999, 110, 6158.

(15) Fast, P. L.; Sanchez, M. L.; Truhlar, D. G. Chem. Phys. Lett. 1999, $306,407$.

(16) Curtiss, L. A.; Redfern, P. C.; Raghavachari, K.; Rassolov, V.; Pople, J. A. J. Chem. Phys. 1999, 110, 4703.

(17) Carroll, D. L. In Developments in Theoretical and Applied Mechanics; Wilson, H., Batara, R., Bert, C., Davis, A., Schapery, R. Stewart, D., Swinson, F., Eds.; School of Engineering, The University of Alabama: Tuscaloosa, AL, 1996; Vol. XVII, p 411.

(18) Microgenetic algorithm calculations were carried out using the program FORTRAN GA, version 1.6.4 as described in: Carroll, D. L. AIAA J. 1996, 34, 338.

(19) Gaudoin, R.; Foulkes, W. M. C. Phys. Rev. B 2002, 66, 052104.

(20) Selected Values of the Thermodynamic Properties of the Elements; Hultgren, R., Desai, P. D., Hawkins, D. T., Gleiser, M., Kelly, K. K., Wagman, D. D., Eds.; American Society for Metals: Metals Park, OH, 1973.

(21) Brewer, L. The Cohesive Energy of the Elements, Report 3720; Lawrence Berkeley Laboratory: Berkeley, CA, 1973.

(22) Erkoç, Ş. Phys. Rep. 1997, 278, 79

(23) Erkoç, Ş. In Annual Reviews of Compuational Physics IX; Stauffer, D., Ed.; World Scientific: Singapore, 2001; pp 1-103.

(24) Duchovic, R. J.; Volobuev, Y. L.; Lynch, G. C.; Jasper, A. W.; Truhlar, D. G.; Allison, T. C.; Wagner, A. F.; Garrett, B. C.; EspinosaGarcía, J.; Corchado, J. C., POTLIB-online, http://comp.chem.umn.edu/ potlib.

(25) Murrell, J. N.; Carter, S.; Farantos, S. C.; Huxley, P.; Varandas, A. J. C. Molecular Potential Energy Functions; Wiley: New York, 1984; p. 29.

(26) Baker, J. A. Phys. Rev. Lett. 1986, 57, 230.

(27) Elrod, M. J.; Saykally, R. J. Chem. Rev. 1994, 94, 1975.

(28) Kostov, M. K.; Cole, M. W.; Lewis, J. C.; Diep, P.; Johnson, J. K. Chem. Phys. Lett. 2000, 332, 26.

(29) Gatica, S. M.; Calbi, M. M.; Cole, M. W.; Velegol, D. Phys. Rev. B 2003, 68, 205409.

(30) Halicioøglu, T.; Pound, G. M. Phys. Stat. Solidi (b) 1975, 30, 619.

(31) Pettifor, D. G.; Ward, M. A. Solid State Commun. 1984, 49, 291.

(32) Harrison, W. A. Elementary Electronic Structure; World Scientific: Singapore, 1999. 

1972.

(33) Torrens, I. M. Interatomic Potentials; Academic Press: New York

(34) de Sainte Claire, P.; Peslherbe, G. H.; Hase, W. L. J. Phys. Chem. 1995, 99, 8147.

(35) Rydberg, R. Z. Phys. 1931, 73, 376

(36) Tang, M. S.; Wang, C. Z.; Chan, C. T.; Ho, K. M. Phys. Rev. B 1996, 53,979 .

(37) Hass, H.; Wang, C. Z.; Fähnle, M.; Elsässer, C.; Ho, K. M. Phys. Rev. B 1998, 57, 1461 .

(38) Nguyen-Mahn, D.; Pettifor, D. G.; Vitek, V. Phys. Rev. Lett. 2000 85,4136 .

(39) Nishitani, S. R.; Ohgushi, S.; Inoue, Y.; Adachi, H. Mater. Sci. Eng. A 2001, 309-310, 490.

(40) Daw, M. S.; Baskes, M. I. Phys. Rev. Lett. 1983, 50, 1285; Phys. Rev. B 1984, 29, 6443.

(41) Ercolessi, F.; Parrinello, M.; Tosatti, E. Philos. Mag. A 1988, 58 , 213

(42) Nishitani, S. R.; Ohgushi, S.; Inoue, Y.; Adachi, H. Mater. Sci. Eng. 2001, A309-310, 490.

(43) Finnis, M. W.; Sinclair, J. E. Philos. Mag. A 1984, 50, 45.

(44) Gupta, R. P. Phys. Rev. B 1985, 23, 6265.

(45) Tomànek, D.; Aligia, A. A.; Balseiro, C. A. Phys. Rev. B 1985, 32,5051 .

(46) Sutton, A. P.; Chen, J. Philos. Mag. Lett. 1990, 61, 139.

(47) Foiles, S. M.; Baskes, M. I.; Daw, M. S. Phys. Rev. B 1986, 33 7983.

(48) Johnson, R. A. Phys. Rev. B 1988, 37, 3924; 1989, 39, 12554; 1990, $41,9717$.

(49) Baskes, M. I. Phys. Rev. B 1992, 46, 2727.

(50) Jacobsen, K. W.; Norskov, J. K.; Puska, M. J. Phys. Rev. B 1987 35,7423 .

(51) Gollisch, H. Surf. Sci. 1986, 166, 87.

(52) Betz, G.; Husinsky, W. Nucl. Instrum. Methods B 1997, 122, 311.
(53) Cleri, F.; Rosato, V. Phys. Rev. B 1993, 48, 22

(54) Papanicolaou, N. I.; Kallinteris, G. C.; Evangelakis, G. A.; Papaconstantopoulos, D. A. Comput. Mater. Sci. 2000, 17, 224

(55) Papanicolaou, N. I.; Chamati, H.; Evangelakis, G. A.; Papaconstantopoulos, D. A. Comput. Mater. Sci. 2003, 27, 191.

(56) Mei, J.; Davenport, J. W. Phys. Rev. B 1992, 46, 21.

(57) Mei, J.; Davenport, J. W.; Fernando, G. W. Phys. Rev. B 1991, 43, 4653.

(58) Streitz F. H.; Mintmire, J. W. Phys. Rev. B 1994, 50, 11996.

(59) Mishin, Y.; Farkas, D.; Mehl, M. J.; Papaconstantopoulos, D. A. Mater. Res. Soc. Symp. Proc. 1999, 538, 535.

(60) Jacobsen, K. W. Com. Condens. Matter Phys. 1988, 14, 129.

(61) Erkoç, Ş. Phys. Stat. Solidi (b) 1989, 152, 447.

(62) Erkoç, Ş. Phys. Stat. Solidi (b) 1989, 155, 461.

(63) Erkoç, Ş. Phys. Stat. Solidi (b) 1990, 161, 211.

(64) Axilrod, B. M.; Teller, E. J. Chem. Phys. 1943, 11, 299

(65) Erkoç, Ş. Dołga (A1) 1985, 9, 203.

(66) Pearson, E.; Takai, T.; Halicioğlu, T.; Tiller, W. A. J. Cryst. Growth 1984, 70, 33.

(67) Murrell J. N.; Mottram, R. E. Mol. Phys. 1990, 69, 571.

(68) Cox, H.; Johnston, R. L.; Murrell, J. N. Surf. Sci. 1997, 373, 67.

(69) Stillinger, F. H.; Weber, T. A. Phys. Rev. B 1985, 31, 5262.

(70) Langhoff, S. R.; Bauschlicher, C. W. Jr. J. Chem. Phys. 1990, 92, 1879.

(71) Fu, Z.; Lemire, G. W.; Bishea, G. A.; Morse, M. D. J. Chem. Phys. 1990, 93, 8420 .

(72) Dewar, M. J. S.; Zoebisch, E. G.; Healy, E. F.; Stewart, J. P. J. Am. Chem. Soc. 1985, 107, 3902.

(73) Dewar, M. J. S.; Holder, A. J. Organometallics 1990, 9, 508.

(74) Dewar, M. J. S.; Thiel, W. J. Am. Chem. Soc. 1977, 99, 4899.

(75) Davis, L. P.; Guirdy, R. M.; Williams, J. R.; Dewar, M. J. S.; Rzepa,

H. S. J. Comput. Chem. 1981, 2, 433. 